\title{
Search for young stars among ROSAT All-Sky Survey X-ray sources in and around the $\mathrm{R}$ CrA dark cloud ${ }^{\star}$
}

\author{
R. Neuhäuser ${ }^{1}$, F.M. Walter ${ }^{2}$, E. Covino ${ }^{3}$, J.M. Alcalá ${ }^{3}$, S.J. Wolk ${ }^{4}$, S. Frink ${ }^{5}$, P. Guillout ${ }^{6}$, M.F. Sterzik ${ }^{7}$, \\ and F. Comerón 8 \\ 1 Max-Planck-Institut für extraterrestrische Physik, D-85740 Garching, Germany \\ e-mail: rne@mpe.mpg.de \\ 2 Department of Physics and Astronomy, SUNY, Stony Brook, NY 11794-3800, U.S.A. \\ 3 Osservatorio Astronomico di Capodimonte, I-80131 Napoli, Italy \\ 4 Harvard-Smithonian Center for Astrophysics, Cambridge, MA 02138, U.S.A. \\ ${ }^{5}$ University of California San Diego, La Jolla, CA 92093, U.S.A. \\ 6 Observatoire Astronomique, CNRS UMR 7550, F-67000 Strasbourg, France \\ 7 European Southern Observatory, Casilla 19001, Santiago 19, Chile \\ 8 European Southern Observatory, Karl-Schwarzschild-Straße 2, D-85748 Garching, Germany
}

Received April 4; accepted August 2, 2000

\begin{abstract}
We present the ROSAT All-Sky Survey data in a $126 \mathrm{deg}^{2}$ area in and around the CrA star forming region. With low-resolution spectroscopy of unidentified ROSAT sources we could find 19 new pre-main sequence stars, two of which are classical $\mathrm{T}$ Tauri stars, the others being weak-lined. The spectral types of these new $\mathrm{T}$ Tauri stars range from F7 to M6. The two new classical $\mathrm{T}$ Tauri stars are located towards two small cloud-lets outside of the main CrA cloud. They appear to be $\sim 10$ Myrs old, by comparing their location in the $\mathrm{H}-\mathrm{R}$ diagram with isochrones for an assumed distance of 130 pc, the distance of the main CrA dark cloud. The new off-cloud weak-line $T$ Tauri stars may have formed in similar cloudlets, which have dispersed recently. High-resolution spectra of our new $\mathrm{T}$ Tauri stars show that they have significantly more lithium absorption than zero-age main-sequence stars of the same spectral type, so that they are indeed young. From those spectra we also obtained rotational and radial velocities. For some stars we found the proper motion in published catalogs. The direction and velocity of the 3D space motion - south relative to the galatic plane - of the CrA T Tauri stars is consistent with the dark cloud being formed originally by a high-velocity cloud impact onto the galactic plane, which triggered

Send offprint requests to: R. Neuhäuser

* Partly based on observations collected at the $1.52 \mathrm{~m}$ and $3.5 \mathrm{~m}$ telescopes of the European Southern Observatory, Chile, in programs 55.E-0549, 57.E-0646, and 63.L-0023, and on observations collected at the $0.9 \mathrm{~m}, 1.5 \mathrm{~m}$, and $4.0 \mathrm{~m}$ CTIO telescope.
\end{abstract}

the star formation in $\mathrm{CrA}$. We also present $V R I J H K$ photometry for most of the new $\mathrm{T}$ Tauri stars to derive their luminosities, ages, and masses.

Key words: stars: formation — stars: luminosiy function, mass function — stars: pre-main sequence - X-rays: stars

\section{Introduction}

The Corona Australis (CrA) molecular cloud complex (Dame et al. 1987) is one of the nearest regions of ongoing and/or recent intermediate- and low-mass star formation. The dark cloud near the emission line star R CrA (Knacke et al. 1973) is the densest cloud core with extinction up to $A_{V} \sim 45 \mathrm{mag}$ (Wilking et al. 1992). This cloud is also called FS 445-47 (Feitzinger \& Stüwe 1984) and condensation $A$ (Rossano 1978); Harju et al. (1993) resolved cloud $A$ into five condensations, the star R CrA being located in A2. Cambrésy (1999) mapped the cloud using optical star counts. Between the stars R and T CrA, there is the reflection nebula NGC 6729; TY CrA and HD 176386 illuminate the nebula NGC 6726/6727. Several infrared (IR) surveys revealed a large population of embedded IR sources (Taylor \& Storey 1984; Wilking et al. 1984, 1986, 1992, 1997), some of which are IR Class I objects, extremely young stars still deeply embedded in their dense circumstellar envelopes (Adams et al. 1987; André $\&$ Montmerle 1994). From the main-sequence contraction time of the early-type stars R, T, and TY CrA, the age of 
the cloud is estimated to be between $\leq 1$ (Knacke et al. 1973) and 6 Myrs (Wilking et al. 1992).

The distance towards the CrA star forming region was estimated by Gaposchkin \& Greenstein (1936) to be $150 \pm 50$ pc and later by Marraco \& Rydgren (1981) to be $\sim 129$ pc (assuming $R=4.5$ ). The Hipparcos satellite tried to measure the parallax of the star $\mathrm{R} \mathrm{CrA}$ and found $122 \pm 68$ mas, i.e. no reliable solution. Casey et al. (1998) estimated the distance towards the eclipsing double-lined spectroscopic binary TY CrA to be $129 \pm 11$ pc from their orbit solution.

Only a few low-mass pre-main sequence (PMS) stars, so-called T Tauri stars (TTS), associated with the CrA dark cloud had been found by $\mathrm{H} \alpha$ and IR surveys (Knacke et al. 1973; Glass \& Penston 1975; Marraco \& Rydgren 1981; Wilking et al. 1984, 1986, 1992, 1997), all being classical TTS (cTTS) with IR excess and strong $\mathrm{H} \alpha$ emission (see Table 1). Patten (1998) obtained optical photometry and spectroscopy of some more sources of, by then, unknown nature around R CrA, previously found by Knacke et al. (1973), Glass \& Penston (1975), and Marraco \& Rydgren (1981), and of some X-ray sources found in a pointed ROSAT observation. He classified some of them as new association members due to $\mathrm{H} \alpha$ emission.

Table 1 gives a list of all previously known optically visible young stars in $\mathrm{Cr}$, with their names, PMS types (Herbig Ae/Be or T Tauri stars), spectral types, $\mathrm{H} \alpha$ and lithium equivalent widths, and some remarks, e.g. on radial velocity and binarity.

With optical follow-up observations of previously unidentified X-ray sources detected with the Einstein Observatory (EO), Walter (1986) and Walter et al. (1997) found eleven new TTS members, namely CrAPMS 1 to 9, two of them (CrAPMS 4 and 6) being visual pairs consisting of two PMS stars (see Table 1). With only one exception (the cTTS CrAPMS 7), all of them are weak-emission line TTS $^{1}$ (wTTS).

In Table 1 we list all the previously known and suspected young stars in $\mathrm{CrA}$, which are optically visible. Walter et al. (1997) could also establish the typical radial velocity of kinematic members of the CrA association: all the seven TTS, for which radial velocities are known, show velocities in the range of -2 to $0 \mathrm{~km} \mathrm{~s}^{-1}$ (heliocentric). Chen et al. (1997) also compiled a list of young stars in CrA and estimated their bolometric luminosities. We omit IR Class I sources and brown dwarf candidates in this pa-

\footnotetext{
${ }^{1}$ Because most wTTS lack the IR excess typical for cTTS and, hence, are probably not surrounded by circumstellar disks, Walter (1986) called them naked TTS. However, because wTTS and nTTS populate both the convective and radiative PMS tracks, the wTTS and nTTS population is different from the so-called post-TTS population. Post-TTS are older than cTTS and do not show signatures of circumstellar disks and accretion $(\mathrm{H} \alpha, \mathrm{UV}, \mathrm{IR})$; post-TTS are expected to exist in large numbers, if star formation has been going on for longer than the typical life-time of circumstellar disks (Herbig 1978).
}

per, because they are too faint in X-rays to be detected in the ROSAT All-Sky Survey (RASS).

The early-type stars TY CrA, HR 7169, and HR 7170, all being spectroscopic binaries, were also detected by EO (Walter et al. 1997), but their X-ray emission may originate from late-type companions. While many of the optically visible TTS are known to be rather strong and variable X-ray emitters (e.g. Montmerle et al. 1983; Walter et al. 1988; Neuhäuser et al. 1995), it was surprising that a few IR Class I objects were also detected by ASCA and ROSAT X-ray observations (Koyama et al. 1996; Neuhäuser \& Preibisch 1997). Wilking et al. (1997) also found five brown dwarf candidates, but they are not detected in deep ROSAT pointings (Neuhäuser et al. 1999).

Because there are several early-type stars in the CrA association, there should be much more than the $\sim 3$ dozen TTS listed in Table 1, if its initial mass function (IMF) is consistent with the Miller-Scalo IMF (Miller \& Scalo 1979). From the spatial incompleteness of the EO observations and the X-ray variability of TTS, Walter et al. (1997) concluded that there should be $\sim 70$ TTS in CrA. From their IR survey, Wilking et al. (1997) estimated the number of the low-mass members to be 22 to 40 for an association age of $\sim 3$ Myrs. If star formation has been ongoing in CrA for more than $\sim 3$ Myrs, there should be even more PMS stars. Such older PMS stars, i.e. the post-TTS, should partly be found around the CrA dark cloud, because they had enough time to disperse out.

Optical follow-up observations of RASS sources in and around other star forming regions (Tau-Aur, Orion, Cha, $\rho$ Oph, Lup-Sco-Cen, etc.) revealed large populations of previously unknown PMS stars most of them being wTTS (see Neuhäuser 1997 for a review), identified as such with low- to intermediate resolution spectroscopy showing late spectral types, $\mathrm{H} \alpha$ emission (or emission filling-in the absorption), and lithium $6708 \AA \mathrm{ab}-$ sorption, a youth indicator. Because some of them were found even outside the star forming clouds, it was argued (Briceño et al. 1997) that many of these young stars are not PMS, but zero-age main-sequence (ZAMS) stars similar to the Pleiades, which also show strong X-ray emission, $\mathrm{H} \alpha$ absorption, and lithium $6708 \AA$ absorption. However, in the meantime, Covino et al. (1997), Neuhäuser et al. (1997), Wichmann et al. (1999), and Alcalá et al. (2000) have shown with high-resolution spectra that most of the previously claimed wTTS really are PMS stars, because they show more lithium than ZAMS stars of the same spectral type. Also, Neuhäuser \& Brandner (1997) found that all 15 Li-rich stars found by ROSAT, which could be placed accurately into the $\mathrm{H}-\mathrm{R}$ diagram using Hipparcos data, clearly are PMS stars. The young stars newly found outside of the clouds could either be ejected out of their parent cloud (Sterzik \& Durisen 1995), or they could have formed locally in small cloud-lets which dispersed since then (Feigelson 1996; Mizuno et al. 1998). Many of the new ROSAT TTS in Taurus, Orion, and 
Table 1. Previously known or suspected optically visible young stars in CrA. PMS types are either cTTS or wTTS or intermediatemass Herbig Ae/Be stars, one being of spectral type F0e. We also list spectral types, H $\alpha$ and lithium $6708 \AA$ equivalent widths (negative when in emission) as well as radial velocities (RV in $\mathrm{km} \mathrm{s}^{-1}$ ), if available. Data for stars with CrAPMS designations are from Walter et al. (1997), for other stars with HBC number from the Herbig-Bell catalog (HBC, Herbig \& Bell 1988), unless otherwise noted. At the end of the table, we also list four more late B-type stars, which might be associated with the CrA cloud. Some of the previously suspected TTS have been confirmed by our spectroscopy, but for MR81 H $\alpha 10$ and Kn anon 2 , we could not detect lithium

\begin{tabular}{|c|c|c|c|c|c|c|c|c|}
\hline $\begin{array}{c}\text { No. } \\
\text { HBC }\end{array}$ & Designation & $\begin{array}{l}\text { GP75 } \\
\text { name }\end{array}$ & Other name & $\begin{array}{l}\text { PMS } \\
\text { type }\end{array}$ & $\begin{array}{l}\text { Spec } \\
\text { type }\end{array}$ & $\begin{array}{r}W_{\lambda}(\mathrm{H} \alpha) \\
{[\AA]}\end{array}$ & $\begin{array}{l}W_{\lambda}(\mathrm{Li}) \\
{[\AA]}\end{array}$ & Remarks \\
\hline 286 & $\mathrm{~S} \mathrm{CrA}$ & & Hen 3-1731 & cTTS & K6 & -90.0 & $0.39^{e}$ & $R V=0,1.37^{\prime \prime}$ binary \\
\hline 287 & TY CrA & & CrAPMS 11 & $\mathrm{HeBe}$ & $\mathrm{B} 9 \mathrm{e}^{a}$ & & yes & triple, $d=129 \mathrm{pc}^{b}$ \\
\hline 288 & $\mathrm{R}$ CrA & & $\mathrm{CoD}-37^{\circ} 13027$ & HeAe & A5e II & & & $R V=-36.0$ \\
\hline 289 & DG $\mathrm{CrA}$ & & Hen 3-1734 & cTTS & $\mathrm{K} 0^{e}$ & $-77.9^{e}$ & $0.57^{e}$ & \\
\hline 290 & $\mathrm{~T} \mathrm{CrA}$ & & & $\mathrm{HeFe}$ & F0e & & & \\
\hline 291 & VV CrA & & Hen 3-1736 & cTTS & $\mathrm{K} 1^{e}$ & $-72.0^{e}$ & & \\
\hline 673 & $\mathrm{MR} 81 \mathrm{H} \alpha 10$ & & & & $\mathrm{~K}$ & $1.0^{e}$ & no ${ }^{e}$ & non-TTS ${ }^{e, g}$ \\
\hline 674 & $\mathrm{MR} 81 \mathrm{H} \alpha 6$ & & CrAPMS 7 & cTTS & M1 & -33.5 & 0.36 & \\
\hline 675 & Kn anon 2 & $\mathrm{j} 2$ & & & $\mathrm{G}^{e}$ & $1.0^{e}$ & $\mathrm{no}^{e}$ & non-TTS ${ }^{e, g}$ \\
\hline 676 & $\mathrm{CoD}-37^{\circ} 13022$ & i2 & CrAPMS 1 & wTTS & K1 & 0.3 & 0.39 & $R V=-1.0$ \\
\hline 677 & $\mathrm{MR} 81 \mathrm{H} \alpha 2$ & $\mathrm{i}$ & HaGr 1-100 & $\operatorname{cTTS}^{e}$ & K8 & $-46.0^{e}$ & $0.47^{e}$ & \\
\hline 678 & V702 CrA & a2 & CrAPMS 2 & wTTS & G5 & -0.7 & 0.28 & $R V=-1.2$ \\
\hline \multirow[t]{2}{*}{679} & CrAPMS 3 & $\mathrm{w}$ & & wTTS & $\mathrm{K} 2$ & -0.9 & 0.41 & $R V=-1.2,4.5^{\prime \prime}$ pair \\
\hline & CrAPMS 3/c & & & wTTS ${ }^{e}$ & $\mathrm{M} 4^{e}$ & $-6.8^{e}$ & $0.36^{e}$ & $4.5^{\prime \prime}$ pair \\
\hline \multirow[t]{24}{*}{680} & $\mathrm{MR} 81 \mathrm{H} \alpha 14$ & & & wTTS $^{e}$ & $\mathrm{M} 3^{e}$ & $-4.6^{e}$ & $0.64^{e}$ & $\mathrm{TTS}^{g}$ \\
\hline & & $\mathrm{e} 2$ & & & $\mathrm{M} 3-5^{g}$ & $\mathrm{em} \cdot{ }^{g}$ & & $\mathrm{TTS}^{g}$ \\
\hline & & $\mathrm{f} 2$ & & & $\mathrm{~K} 4^{g}$ & & & $\mathrm{TTS}^{g}$ \\
\hline & & $\mathrm{n}$ & & $\mathrm{cTTS}^{g}$ & & $\mathrm{em} \cdot{ }^{g}$ & & \\
\hline & MR81 H $\alpha 12$ & & & & M3- $5^{g}$ & em..$^{g}$ & & $\mathrm{TTS}^{g}$ \\
\hline & $\mathrm{MR} 81 \mathrm{H} \alpha 13$ & & & & $\mathrm{M} 3-5^{g}$ & em. ${ }^{g}$ & & $\mathrm{TTS}^{g}$ \\
\hline & $\mathrm{MR} 81 \mathrm{H} \alpha 15$ & & & & $\mathrm{M} 3-5^{g}$ & em..$^{g}$ & & $\mathrm{TTS}^{g}$ \\
\hline & $\mathrm{MR} 81 \mathrm{H} \alpha 16$ & & & & $\mathrm{M}^{g}$ & $\mathrm{em} \cdot{ }^{g}$ & & $\mathrm{TTS}^{g}$ \\
\hline & $\mathrm{MR} 81 \mathrm{H} \alpha 17$ & & & & $\mathrm{M} 3-5^{g}$ & $\mathrm{em} .^{g}$ & & $\mathrm{TTS}^{g}$ \\
\hline & CrAPMS $4 \mathrm{NW}$ & & & wTTS & M0.5 & -1.1 & 0.45 & $R V=-2.2$ \\
\hline & CrAPMS 4SE & & & wTTS & G5 & 1.0 & 0.36 & $R V=-2.0$ \\
\hline & CrAPMS 5 & & & wTTS & K5 & -0.8 & 0.44 & $R V=-0.8$ \\
\hline & $\mathrm{MR} 81 \mathrm{H} \alpha 11 \mathrm{NE}$ & & CrAPMS $6 \mathrm{NE}$ & wTTS & M3 & -5.9 & 0.70 & $\mathrm{NE} / \mathrm{SW} 3^{\prime \prime}$ pair \\
\hline & MR81 H $\alpha$ 11SW & & CrAPMS 6SW & wTTS & M3.5 & -9.8 & 0.44 & $\mathrm{NE} / \mathrm{SW} 3^{\prime \prime}$ pair \\
\hline & CrAPMS 8 & g2 & Patten R9 & wTTS & M3 & -3.9 & 0.57 & \\
\hline & CrAPMS 9 & & & wTTS & M2 & -9.2 & 0.5 & \\
\hline & RXJ1855.1-3754 & & GSC 07916-00050 & $\mathrm{wTTS}^{d}$ & $K 3^{d}$ & $1.6^{d}$ & $0.38^{d}$ & $W(\mathrm{Li}) \simeq W(\mathrm{Ca})^{d}$ \\
\hline & RXJ1857.7-3719 & & Patten R1c & & $\mathrm{M} 3-5^{g}$ & $\mathrm{em} \cdot{ }^{g}$ & & $\mathrm{TTS}^{g}$ \\
\hline & RXJ1858.9-3640 & & Patten R17c & & $\mathrm{M} 3-5^{g}$ & $\mathrm{em} \cdot{ }^{g}$ & & $\mathrm{TTS}^{g}$ \\
\hline & RXJ1859.7-3655 & & Patten R13a & & $\mathrm{M}^{g}$ & em. ${ }^{g}$ & & $\mathrm{TTS}^{g}$ \\
\hline & HR 7169 & l & HD 176269 & & B9 & $7.7^{e}$ & & $d \simeq 134 \mathrm{pc}^{f}(*)$ \\
\hline & HR 7170 & $\mathrm{k}$ & HD 176270 & & $\mathrm{~B} 8$ & $7.0^{e}$ & & $d \simeq 77 \mathrm{pc}^{f}(*)$ \\
\hline & SAO 210888 & & HD 177076 & & B9.5 & & & $d \simeq 185 \mathrm{pc}^{f}$ \\
\hline & HD 176386 & $\mathrm{p}$ & HIP 93425 & & B9 & & $V=7.3^{c}$ & $d \simeq 136 \mathrm{pc}^{f}, 4^{\prime \prime}$ binary $^{c}$ \\
\hline
\end{tabular}

References: (a) HBC, (b) Casey et al. 1998, (c) Simbad, (d) Neuhäuser et al. 1997, (e) this work, (f) Hipparcos, (g) Patten 1998. Note: (*) Both HR 7169 and HR 7170 are spectroscopic binaries (Hoffleit 1982). With a separation of $13^{\prime \prime}$ in 1982.66 (Torres 1985), this visual pair may be bound. The system was also detected by EO as the spatially unresolved source PMSCrA 10 (Walter et al. 1997). 
Lup-Sco-Cen are probably members of the Gould Belt (Guillout et al. 1998a,b), young stars still at least slightly above the ZAMS.

The CrA dark cloud is located $\sim 18^{\circ}$ below the galactic plane. According to Olano (1982, see also Fig. 1.10 in Pöppel 1997), the CrA and Chamaeleon clouds are not part of the Gould Belt nor of the Lindblad ring, because both $\mathrm{CrA}$ and Cha are far below the galactic plane, while the belt and the ring are both above the plane. In the cross-correlation of Tycho and RASS, the CrA association is seen as a small cluster of X-ray active stars around $l \simeq 0^{\circ}$ and $b \simeq-20^{\circ}$, see Fig. 3 in Guillout et al. (1998a) and Fig. 3 in Guillout et al. (1998b). The Gould Belt is above the galactic plane in this quadrant. Hence, neither CrA nor Cha are part of the belt, so that we should not expect to detect Gould Belt members in CrA. Many new TTS were discovered around the Cha clouds (Alcalá et al. 1995; Covino et al. 1997), so that we may expect to find many such TTS also here around the CrA dark cloud. Hence, we carried out an optical identification program to find more PMS in and around the CrA dark cloud among unidentified RASS sources ${ }^{2}$. In Sect. 2, we describe the X-ray data reduction and list all X-ray sources found with RASS (Table 2). Our spectra are presented in Sect. 3 together with lists of potential optical counterparts (Table 3). Then, in Sect. 4, we discuss the results of the spectroscopy including Table 4 with our new TTS. In Sect. 5 we list the available optical and IR photometry for the new TTS; the $\mathrm{H}-\mathrm{R}$ diagram is shown and discussed in Sect. 6. Then, in Sect. 7, we present proper motions of some of our new PMS stars and discuss the 3D space motion of young stars in CrA. Finally, in Sect. 8, we estimate the completeness of our survey. We summarize our results in the last section.

\section{X-ray data reduction}

The previously known young stars associated with the CrA dark cloud are located in a small, $\sim 126 \mathrm{deg}^{2}$ area around R CrA near $\alpha_{2000}=19^{\mathrm{h}}$ and $\delta_{2000}=-37^{\circ}$. Because we also expect to find previously unknown young

\footnotetext{
${ }^{2}$ Four new PMS stars were already identified among ROSAT sources:

GSC 07916-00050 as the optical counterpart to RXJ1855.13754 (Neuhäuser et al. 1997), an X-ray source found with the ROSAT High Resolution Imager (HRI) in a deep pointed observation centered on RXJ1856.5-3754, an isolated radio-quiet neutron star (Walter et al. 1996; Walter \& Matthews 1997). Neuhäuser et al. (1997) identified GSC 07916-00050 in order to confirm that it is the true (i.e. possibly X-ray emitting) counterpart to RXJ1855.1-3754, to be able to perform a correct boresight correction of the position of the central HRI source. In addition, Patten (1998) identified three new PMS stars among X-ray sources found in a pointed observation with the ROSAT Positional Sensitive Proportional Counter (PSPC), also listed in Table 1.
}

stars around the dark cloud, we investigated the area $\alpha_{2000}=18^{\mathrm{h}} 35^{\mathrm{m}}$ to $19^{\mathrm{h}} 38^{\mathrm{m}}$ and $\delta_{2000}=-41^{\circ}$ to $-33^{\circ}$.

We reduced all RASS II data ${ }^{3}$ pertaining to that area with the Extended Scientific Analysis Software (EXSAS, Zimmermann et al. 1994) version 98APR running under ESO-MIDAS version $97 \mathrm{NOV}$. We performed standard local and map source detection in the bands soft $(0.1$ to $0.4 \mathrm{keV})$, hard 1 ( 0.5 to $0.9 \mathrm{keV})$, hard 2 (0.9 to $2.0 \mathrm{keV})$, hard ( 0.5 to $2.0 \mathrm{keV})$, and broad $(0.1$ to $2.4 \mathrm{keV})$, as described in detail in Neuhäuser et al. (1995). After merging the source lists, each source was again tested in the above mentioned five bands by a maximum likelihood source detection algorithm. Following Neuhäuser et al. (1995) we accept only sources with $M L \geq 7.4$ corresponding to $\sim 3.5 \sigma$ as real.

The moderate energy resolution of the PSPC allows to extract some spectral information from the RASS data, namely so-called hardness ratios, i.e., X-ray colors, which are defined as follows: If $Z_{s, h 1, h 2}$ are the count rates in the bands soft, hard 1, and hard 2, respectively, then

$H R 1=\frac{Z_{h 1}+Z_{h 2}-Z_{s}}{Z_{h 1}+Z_{h 2}+Z_{s}} \quad \& \quad H R 2=\frac{Z_{h 2}-Z_{h 1}}{Z_{h 2}+Z_{h 1}} \cdot(1)$ I.e., hardness ratios range from -1 to +1 , hardness ratio errors can be larger than 2. If no counts are detected, e.g, in the soft band, then $H R 1=1$, but one can estimate a lower limit to $H R 1$ by using the upper limit to the soft band count rate $Z_{\mathrm{s}}$ in Eq. (1), similar for upper limits to HR 1 as well as for upper and lower limits to $H R 2$.

In Table 2, we list all RASS X-ray sources detected in our study area with X-ray position, likelihood for existence, hardness ratio, number of counts, exposure time, and individual energy conversion factor

$E C F=(5.30 \cdot H R 1+8.31) \cdot 10^{-12} \mathrm{erg} \mathrm{cm}^{-2} \mathrm{cts}^{-1}$

according to Schmitt et al. (1995).

In Table 3, we list the optical counterparts to the X-ray sources, most of which are the closest counterparts, but in four cases, the most likely counterpart turned out to be not the closest potential optical counterpart. Table 3 gives optical positions, offsets between X-ray and optical positions, $V$ magnitudes, $\mathrm{X}$-ray to optical flux ratios, identification information, e.g. whether it is a new young star, and remarks, e.g. on previously known stars found among our X-ray sources. The typical positional error of RASS sources is 40 arcsec (Neuhäuser et al. 1995), which we allow as offset between optical and X-ray position for source identification. We searched for stellar counterparts in Simbad, the Hubble Space Telescope Guide Star Catalog (GSC), and the NASA Extragalactic Database (NED). We list in Table 3 the optical positions,

\footnotetext{
${ }^{3}$ Prior to the optical follow-up observations, we reduced the earlier version of the ROSAT data (RASS I) with an earlier version of EXSAS; although the source lists and X-ray properties are sightly different, all newly identified young stars are detected in both RASS I and RASS II.
} 
Table 2. X-ray data for our sample. Listed are all X-ray sources detected in the ROSAT All-Sky Survey sorted by right ascension. We list a running number (used in other tables for cross-reference), the ROSAT source designation, the X-ray source position (J2000.0), the maximum likelihood of existence $M L$, the hardness ratios with errors, the (background subtracted and vignetting corrected) number of counts in the broad band ( 0.1 to $2.4 \mathrm{keV})$ with errors, the exposure time in seconds, and log $E C F$ for the energy conversion factor in counts $\mathrm{erg}^{-1} \mathrm{~cm}^{2}$

\begin{tabular}{|c|c|c|c|c|c|c|c|c|c|}
\hline No. & Designation & $\alpha_{2000(\mathrm{X}}$ & (-ray) $\delta_{2000}$ & $M L$ & $H R 1$ & $H R 2$ & Counts & Exp. [s] & og $E C F$ \\
\hline 1 & RXJ1835.1-3404 & $18: 35: 08.0$ & $-34: 04: 20.0$ & 10.2 & $\geq 0.61$ & $0.30 \pm 0.36$ & $8.9 \pm 4.0$ & 147.2 & -10.87 \\
\hline 2 & RXJ1835.3-3927 & $18: 35: 21.5$ & $-39: 27: 13.3$ & 8.1 & $5 \pm 0.49$ & $\geq 0.10$ & $7.1 \pm 3.2$ & 124.3 & -11.04 \\
\hline 3 & RXJ1835.7-3259 & $18: 35: 47.0$ & $-32: 59: 41.5$ & 1240.1 & $3 \pm 0.02$ & $0.53 \pm 0.05$ & $364.0 \pm 19.6$ & 163.0 & -10.88 \\
\hline 4 & RXJ1835.8-3813 & $18: 35: 49.2$ & $-38: 13: 24.1$ & 9.8 & $77 \pm 0.41$ & $0.02 \pm 0.44$ & $11.7 \pm 4.3$ & 139.4 & -10.91 \\
\hline 5 & RXJ1835.8-4046 & $18: 35: 53.4$ & $-40: 46: 23.5$ & 10.2 & $\geq 0.27$ & $\geq 0.43$ & $3.6 \pm 2.3$ & 125.7 & -10.87 \\
\hline 6 & RXJ1835.9-3525 & $18: 35: 58.3$ & $-35: 25: 35.5$ & 7.7 & $0.77 \pm 0.44$ & $\geq 0.42$ & $6.1 \pm 3.4$ & 143.3 & -10.91 \\
\hline 7 & RXJ1836.3-4010 & $18: 36: 18.7$ & $-40: 10: 20.2$ & 13.1 & $-0.65 \pm 0.40$ & $\leq 0.19$ & $11.2 \pm 4.0$ & 131.5 & -11.31 \\
\hline 8 & RXJ1836.6-3451 & $18: 36: 40.6$ & $-34: 51: 33.5$ & 13.2 & $\geq 0.44$ & $0.28 \pm 0.42$ & $9.4 \pm 3.9$ & 139.1 & -10.87 \\
\hline 9 & RXJ1837.3-3442 & $18: 37: 18.2$ & $-34: 42: 39.0$ & 13.1 & $0.25 \pm 0.40$ & $0.05 \pm 0.54$ & $9.2 \pm 3.5$ & 133.2 & -11.02 \\
\hline 10 & RXJ1838.2-3838 & $18: 38: 17.1$ & $-38: 38: 12.9$ & 11.8 & $\geq 0.47$ & $0.31 \pm 0.41$ & $8.0 \pm 3.3$ & 142.1 & -10.87 \\
\hline 11 & RXJ1838.3-3523 & $18: 38: 20.2$ & $-35: 23: 32.6$ & 10.3 & $-0.67 \pm 0.52$ & $\leq 0.42$ & $9.4 \pm 3.8$ & 140.0 & -11.32 \\
\hline 12 & RXJ1839.0-3705 & 18:39:05.8 & $-37: 05: 46.8$ & 21.6 & $0.84 \pm 0.24$ & $-0.03 \pm 0.31$ & $13.4 \pm 4.2$ & 139.0 & -10.89 \\
\hline 13 & RXJ1839.0-3726 & 18:39:05.9 & $-37: 26: 36.0$ & 12.3 & $0.78 \pm 0.21$ & $-0.30 \pm 0.39$ & $9.7 \pm 3.9$ & 142.1 & -10.90 \\
\hline 14 & RXJ1839.2-3854 & $18: 39: 16.1$ & $-38: 54: 13.8$ & 9.2 & $\geq 0.18$ & $\geq 0.40$ & $3.3 \pm 2.5$ & 135.4 & -10.87 \\
\hline 15 & RXJ1839.7-3458 & 18:39:46.8 & $-34: 58: 58.2$ & 7.8 & $\geq 0.55$ & $0.51 \pm 0.35$ & $5.8 \pm 3.2$ & 167.4 & -10.87 \\
\hline 16 & RXJ1840.1-3640 & 18:40:09.5 & $-36: 40: 09.1$ & 7.9 & $0.24 \pm 0.40$ & $0.08 \pm 0.52$ & $8.6 \pm 3.6$ & 154.6 & -11.02 \\
\hline 17 & $.6-3728$ & $18: 40$ & $-37: 28: 44.3$ & 13.0 & $\geq 0.67$ & $0.29 \pm 0.36$ & $8.6 \pm 3.6$ & 150.6 & -10.87 \\
\hline 18 & RXJ1840.6-3612 & $18: 40: 39.7$ & $-36: 12: 14.1$ & 7.8 & $0.34 \pm 0.37$ & $-0.01 \pm 0.48$ & $11.1 \pm 4.2$ & 150.4 & -10.99 \\
\hline 19 & RXJ1840.8-3547 & $18: 40: 53.8$ & $-35: 47: 06.2$ & 32.7 & $-0.13 \pm 0.25$ & $-0.19 \pm 0.38$ & $20.2 \pm 5.0$ & 147.3 & -11.12 \\
\hline 20 & RXJ1840.9-3350 & $18: 40: 55.1$ & $-33: 50: 52.5$ & 14.9 & $0.28 \pm 0.30$ & $0.38 \pm 0.33$ & $16.4 \pm 5.1$ & 180.2 & -11.01 \\
\hline 21 & $.2-3821$ & 13.5 & $-38: 21: 55.9$ & 9.1 & $\leq-0.49$ & & $5.7 \pm 3.1$ & 51.9 & -11.52 \\
\hline 22 & RXJ1841.5-3508 & $18: 41: 34.2$ & $-35: 08: 27.7$ & 11.2 & $0.15 \pm 0.38$ & $-0.28 \pm 0.53$ & $9.5 \pm 3.8$ & 154.7 & -11.04 \\
\hline 23 & RXJ1841.8-3525 & $18: 41: 49.7$ & $-35: 25: 44.3$ & 58.2 & $0.47 \pm 0.18$ & $-0.14 \pm 0.24$ & $26.6 \pm 5.6$ & 140.0 & -10.97 \\
\hline 24 & RXJ1842.1-3732 & $18: 42: 12.0$ & $-37: 32: 33.0$ & 9.4 & $0.05 \pm 0.38$ & $-0.09 \pm 0.53$ & $9.7 \pm 3.8$ & 141.1 & -11.07 \\
\hline 25 & -3845 & $18: 4$ & $-38: 45: 28.3$ & 8.7 & $\geq 0.40$ & $0.46 \pm 0.45$ & $8.7 \pm 3.6$ & 137.4 & -10.87 \\
\hline 26 & -3451 & $18: 4$ & $-34: 51: 00.3$ & 7.6 & $5 \pm 0.50$ & $\geq 0.22$ & $3.7 \pm 2.4$ & 154.3 & -10.87 \\
\hline 27 & RXJ1842.9-3532 & $18: 42: 58.4$ & $-35: 32: 34.6$ & 21.7 & $0.90 \pm 0.24$ & $0.29 \pm 0.34$ & $10.9 \pm 3.9$ & 135.3 & -10.88 \\
\hline 28 & RXJ1843.0-3331 & $18: 43: 05.2$ & $-33: 31: 07.6$ & 8.1 & $0.35 \pm 0.40$ & $0.14 \pm 0.49$ & $8.7 \pm 3.9$ & 191.5 & -10.99 \\
\hline 29 & RXJ1843.4-3314 & $18: 43: 24.5$ & $-33: 14: 31.4$ & 11.8 & $-0.51 \pm 0.35$ & $\leq 0.32$ & $14.3 \pm 5.0$ & 194.2 & -11.25 \\
\hline 30 & 3541 & 18: & $-35: 41: 43.5$ & 30.0 & \pm 0.19 & $0.12 \pm 0.26$ & $24.5 \pm 5.8$ & 149.5 & -10.92 \\
\hline 31 & $.5-3723$ & $18: 4$ & $-37: 23: 59.8$ & 43.4 & $0.62 \pm 0.20$ & $0.18 \pm 0.26$ & $23.2 \pm 5.2$ & 141.1 & -10.94 \\
\hline 32 & RXJ1844.5-3739 & $18: 44: 31.3$ & $-37: 39: 13.9$ & 10.4 & $0.53 \pm 0.46$ & $\geq 0.24$ & $6.9 \pm 3.3$ & 134.9 & -10.95 \\
\hline 33 & RXJ1844.7-3313 & $18: 44: 43.4$ & $-33: 13: 56.1$ & 8.9 & $0.83 \pm 0.31$ & $0.78 \pm 0.47$ & $7.4 \pm 3.8$ & 206.1 & -10.90 \\
\hline 34 & -3450 & $18: 4$ & $-34: 50: 55.2$ & 7.9 & $8 \pm 0.65$ & $\geq 0.41$ & $4.8 \pm 2.9$ & 185.1 & -10.92 \\
\hline 35 & RXJ1845.1-3324 & $18: 45$ & $-33: 24: 26.3$ & 10.6 & $-0.28 \pm 0.38$ & $0.20 \pm 0.62$ & $9.6 \pm 3.9$ & 200.1 & -11.16 \\
\hline 36 & RXJ1845.5-3750 & $18: 45: 34.4$ & $-37: 50: 24.3$ & 57.7 & $0.59 \pm 0.15$ & $0.28 \pm 0.18$ & $39.6 \pm 6.9$ & 143.7 & -10.94 \\
\hline 37 & RXJ1846.3-3552 & $18: 46: 18.9$ & $-35: 52: 34.0$ & 7.9 & $\leq-0.34$ & & $5.6 \pm 3.0$ & 161.1 & -11.52 \\
\hline 38 & $6.7-3604$ & $18: 46: 44.3$ & $-36: 04: 40.4$ & 8.0 & $\geq-0.09$ & $0.06 \pm 0.59$ & $5.1 \pm 2.9$ & 143.7 & -10.87 \\
\hline 39 & RXJ1846.7-3636 & $18: 46: 46.2$ & $-36: 36: 21.3$ & 29.2 & $0.07 \pm 0.27$ & $0.20 \pm 0.36$ & $19.1 \pm 4.9$ & 142.0 & -11.06 \\
\hline 40 & RXJ1846.8-3911 & $18: 46: 53.3$ & $-39: 11: 10.3$ & 7.8 & $0.45 \pm 0.42$ & $-0.12 \pm 0.49$ & $8.9 \pm 3.6$ & 125.3 & -10.97 \\
\hline 41 & RXJ1846.9-3503 & $18: 46: 58.0$ & $-35: 03: 59.4$ & 9.1 & $0.84 \pm 0.33$ & $\leq-0.59$ & $7.9 \pm 3.8$ & 176.3 & -10.89 \\
\hline 42 & RXJ1847.2-3709 & $18: 47: 14.3$ & $-37: 09: 43.3$ & 8.6 & $0.47 \pm 0.42$ & $\leq-0.30$ & $6.5 \pm 3.2$ & 136.3 & -10.97 \\
\hline 43 & RXJ1847.5-3642 & $18: 47: 32.9$ & $-36: 42: 43.9$ & 9.6 & $0.93 \pm 0.44$ & $0.13 \pm 0.52$ & $4.6 \pm 2.3$ & 136.0 & -10.88 \\
\hline 44 & RXJ1847.7-3606 & $18: 47: 46.5$ & $-36: 06: 12.4$ & 29.7 & $\geq 0.68$ & $0.14 \pm 0.28$ & $15.7 \pm 4.4$ & 154.3 & -10.87 \\
\hline 45 & RXJ1847.7-4024 & $18: 47: 46.9$ & $-40: 24: 14.2$ & 69.8 & $0.00 \pm 0.19$ & $0.35 \pm 0.24$ & $36.7 \pm 6.5$ & 130.2 & -11.08 \\
\hline 46 & RXJ1847.8-3828 & $18: 47: 51.9$ & $-38: 28: 45.9$ & 7.4 & $\geq 0.19$ & $\geq 0.23$ & $2.2 \pm 1.7$ & 122.5 & -10.87 \\
\hline 47 & RXJ1848.2-3415 & $18: 48: 12.1$ & $-34: 15: 41.3$ & 8.2 & $0.42 \pm 0.39$ & $-0.16 \pm 0.47$ & $9.4 \pm 4.0$ & 202.0 & -10.98 \\
\hline 48 & RXJ1848.6-3458 & $18: 48: 36.1$ & $-34: 58: 58.0$ & 8.0 & $0.08 \pm 0.59$ & $0.22 \pm 0.85$ & $8.7 \pm 3.8$ & 200.2 & -11.06 \\
\hline 49 & RXJ1849.0-3734 & $18: 49: 02.0$ & $-37: 34: 22.7$ & 9.6 & $\geq 0.40$ & $-0.44 \pm 0.41$ & $6.0 \pm 3.0$ & 157.8 & -10.87 \\
\hline 50 & RXJ1849.1-3546 & 18:49:08.7 & $-35: 46: 41.7$ & 45.0 & $0.71 \pm 0.17$ & $0.56 \pm 0.20$ & $22.5 \pm 5.2$ & 193.1 & -10.92 \\
\hline 51 & $.4-3455$ & $18: 51: 26.1$ & $-34: 55: 31.6$ & 8.1 & $\geq 0.39$ & $-0.45 \pm 0.48$ & $5.3 \pm 3.0$ & 211.0 & -10.87 \\
\hline 52 & RXJ1852.1-3607 & $18: 52: 10.2$ & $-36: 07: 24.9$ & 7.8 & $0.19 \pm 0.39$ & $-0.57 \pm 0.43$ & $9.4 \pm 3.8$ & 171.9 & -11.03 \\
\hline
\end{tabular}


Table 2. continued

\begin{tabular}{|c|c|c|c|c|c|c|c|c|c|}
\hline No. & Designation & $\alpha_{2000}(\mathrm{X}$ & -ray) $\delta_{2000}$ & $M L$ & $H R 1$ & $H R 2$ & Counts & Exp. [s] & $\log E C F$ \\
\hline 53 & RXJ1852.3-3700 & $18: 52: 18.2$ & $-37: 00: 20.4$ & 36.3 & $0.90 \pm 0.13$ & $0.54 \pm 0.25$ & $14.7 \pm 4.3$ & 155.5 & -10.88 \\
\hline 54 & RXJ1852.4-3730 & $18: 52: 25.6$ & $-37: 30: 32.4$ & 54.4 & $-0.41 \pm 0.19$ & $-0.26 \pm 0.38$ & $29.4 \pm 6.0$ & 152.8 & -11.21 \\
\hline 55 & XJ1853.1-3609 & $18: 53: 06.5$ & $-36: 09: 54.7$ & 55.5 & $0.33 \pm 0.18$ & $0.40 \pm 0.22$ & $37.1 \pm 6.8$ & 190.8 & -11.00 \\
\hline 56 & RXJ1853.4-4020 & $18: 53: 26.3$ & $-40: 20: 02.8$ & 9.2 & $-0.18 \pm 0.49$ & $\leq 0.01$ & $7.7 \pm 3.4$ & 147.6 & -11.13 \\
\hline 57 & RXJ1853.5-3544 & $18: 53: 36.0$ & $-35: 44: 32.7$ & 9.4 & $\geq 0.34$ & $0.10 \pm 0.48$ & $6.4 \pm 3.3$ & 214.1 & -10.87 \\
\hline 58 & XJ1854.4-4010 & $18: 54: 25.7$ & $-40: 10: 21.7$ & 9.3 & $\geq 0.32$ & $0.40 \pm 0.48$ & $7.1 \pm 3.4$ & 154.8 & -10.87 \\
\hline 59 & XJ1854.4-3738 & $18: 54: 28.2$ & $-37: 38: 39.7$ & 7.7 & $\geq 0.37$ & $0.39 \pm 0.44$ & $6.0 \pm 3.1$ & 173.8 & -10.87 \\
\hline 60 & XJ1854.5-3823 & 18:54:34.3 & $-38: 23: 22.2$ & 8.5 & $-0.31 \pm 0.42$ & $\leq-0.27$ & $13.0 \pm 4.6$ & 161.7 & -11.18 \\
\hline 61 & RXJ1854.9-3600 & $18: 54: 59.4$ & $-36: 00: 26.0$ & 8.9 & $\geq 0.40$ & $-0.47 \pm 0.44$ & $9.5 \pm 4.0$ & 232.8 & -10.87 \\
\hline 62 & RXJ1855.5-3806 & $18: 55: 32.5$ & $-38: 06: 41.2$ & 12.7 & $\geq 0.58$ & $0.76 \pm 0.32$ & $8.4 \pm 4.0$ & 171.9 & -10.87 \\
\hline 63 & XJ1856.5-3754 & $18: 56: 35.1$ & $-37: 54: 32.7$ & 2643.8 & $2 \pm 0.02$ & $-0.94 \pm 0.16$ & $637.3 \pm 25.5$ & 166.8 & -11.46 \\
\hline 64 & 3545 & 43.7 & $-35: 45: 23.1$ & 8.9 & $\geq 0.07$ & $0.22 \pm 0.28$ & $4.7 \pm 2.7$ & 216.0 & -10.87 \\
\hline 65 & XJ1856.7-4021 & $18: 56: 46.2$ & $-40: 21: 10.7$ & 12.0 & $\geq 0.52$ & $0.61 \pm 0.37$ & $7.0 \pm 3.5$ & 166.0 & -10.87 \\
\hline 66 & RXJ1857.3-3635 & $18: 57: 19.8$ & $-36: 35: 49.3$ & 10.6 & $-0.45 \pm 0.38$ & $-0.78 \pm 1.33$ & $11.4 \pm 4.2$ & 211.7 & -11.23 \\
\hline 67 & RXJ1857.3-3554 & $18: 57: 22.4$ & $-35: 54: 19.4$ & 10.0 & $\leq-0.45$ & $\geq-0.65$ & $6.7 \pm 3.3$ & 244.5 & -11.52 \\
\hline 68 & 3732 & $18:$ & $-37: 32: 57.3$ & 10.5 & $0.78 \pm 0.37$ & $0.95 \pm 0.37$ & $5.8 \pm 2.9$ & 176.8 & -10.90 \\
\hline 69 & 651 & 0.1 & $-36: 51: 10.7$ & 8.5 & $\leq-0.43$ & $\geq-0.83$ & $8.3 \pm 3.8$ & 225.4 & -11.52 \\
\hline 70 & RXJ1858.0-3640 & $18: 58: 05.3$ & $-36: 40: 57.2$ & 8.1 & $-0.01 \pm 0.41$ & $-0.44 \pm 0.47$ & $10.3 \pm 4.3$ & 236.1 & -11.08 \\
\hline 71 & RXJ1858.4-3446 & $18: 58: 29.4$ & $-34: 46: 53.2$ & 32.1 & $0.83 \pm 0.16$ & $-0.32 \pm 0.23$ & $27.8 \pm 6.4$ & 312.3 & -10.90 \\
\hline 72 & -3630 & 40.4 & $-36: 30: 38.8$ & 12.3 & $-0.17 \pm 0.35$ & $0.42 \pm 0.45$ & $12.5 \pm 4.6$ & 257.0 & -11.13 \\
\hline 73 & 3706 & 3.5 & $-37: 06: 18.3$ & 25.1 & $-0.19 \pm 0.27$ & $0.22 \pm 0.49$ & $20.9 \pm 5.3$ & 229.4 & -11.14 \\
\hline 74 & $9.0-4051$ & 01.1 & $-40: 51: 19.5$ & 8.5 & $\geq 0.14$ & $\leq-0.23$ & $6.0 \pm 3.2$ & 154.6 & -10.87 \\
\hline 75 & RXJ1859.3-3406 & $18: 59: 21.7$ & $-34: 06: 09.9$ & 7.5 & $\geq 0.55$ & $-0.16 \pm 0.42$ & $6.1 \pm 3.7$ & 300.1 & -10.87 \\
\hline 76 & RXJ1900.6-3810 & 19:00:38.9 & $-38: 10: 19.5$ & 14.2 & $0.99 \pm 0.15$ & $0.27 \pm 0.30$ & $13.4 \pm 4.9$ & 234.9 & -10.87 \\
\hline 77 & 53 & 1.4 & -34 & 7.9 & $0.99 \pm 0.44$ & $0.10 \pm 0.39$ & $9.9 \pm 4.3$ & 296.8 & -10.87 \\
\hline 78 & 928 & 19: & $-39: 2$ & 10.3 & $\geq 0.54$ & $-0.43 \pm 0.35$ & $8.1 \pm 3.8$ & 190.4 & -10.87 \\
\hline 79 & 3333 & 19:0 & $-33: 33: 40.8$ & 8.9 & $\geq 0.39$ & $\leq-0.35$ & $3.6 \pm 2.4$ & 233.9 & -10.87 \\
\hline 80 & RXJ1901.1-3648 & 19:01:09.2 & $-36: 48: 01.7$ & 9.8 & $0.42 \pm 0.39$ & $0 . \overline{4} \pm 0.42$ & $9.7 \pm 4.0$ & 272.1 & -10.98 \\
\hline 81 & $.4-4022$ & $19: 0$ & $-40: 22: 31.9$ & 11.3 & $0.63 \pm 0.63$ & $-0.17 \pm 0.46$ & $11.0 \pm 4.2$ & 188.3 & -10.93 \\
\hline 82 & 3422 & 26.8 & $-34: 22: 44.8$ & 48.4 & $0.11 \pm 0.19$ & $-0.40 \pm 0.23$ & $37.7 \pm 7.1$ & 295.0 & -11.05 \\
\hline 83 & RXJ1901.5-3700 & 19:0 & $-37: 00: 55.1$ & 37.7 & $0.83 \pm 0.17$ & $-0.06 \pm 0.22$ & $26.8 \pm 6.1$ & 268.0 & -10.90 \\
\hline 84 & XJ1901.6-3652 & 19:01:38.9 & $-36: 52: 55.0$ & 38.3 & $0.80 \pm 0.18$ & $0.54 \pm 0.20$ & $24.6 \pm 5.8$ & 278.8 & -10.90 \\
\hline 85 & RXJ1901.6-3644 & 19:01:39.0 & $-36: 44: 52.4$ & 27.1 & $\geq 0.61$ & $0.95 \pm 0.15$ & $15.2 \pm 4.8$ & 279.1 & -10.87 \\
\hline 86 & 8822 & 3.1 & $-38: 22: 02.4$ & 8.9 & $0.15 \pm 0.42$ & $\geq 0.21$ & $8.3 \pm 3.5$ & 237.5 & -11.04 \\
\hline 87 & RXJ1902.0 & 03.1 & $-37: 07: 47.7$ & 22.6 & $0.71 \pm 0.21$ & $0.58 \pm 0.24$ & $17.5 \pm 4.8$ & 268.1 & -10.92 \\
\hline 88 & RXJ1902.3-3310 & $19: 02: 20.0$ & $-33: 10: 45.8$ & 8.0 & $-0.82 \pm 0.47$ & & $8.1 \pm 3.7$ & 187.4 & -11.40 \\
\hline 89 & RXJ1902.3-3655 & $19: 02: 22.7$ & $-36: 55: 42.8$ & 7.5 & $\geq 0.07$ & $0.76 \pm 0.38$ & $4.8 \pm 2.8$ & 282.5 & -10.87 \\
\hline 90 & 3922 & 2.8 & $-39: 22: 11.2$ & 9.5 & $0.32 \pm 0.44$ & $-0.64 \pm 0.85$ & $9.4 \pm 3.8$ & 204.3 & -11.18 \\
\hline 91 & XJ & 19:0 & $-36: 17$ & 7.6 & $\geq 0.54$ & $-0.06 \pm 0.48$ & $4.1 \pm 2.7$ & 306.9 & -10.87 \\
\hline 92 & RXJ1902.5-3504 & 19:02:31.6 & $-35: 04: 58.8$ & 9.5 & $\leq-0.58$ & & $7.0 \pm 3.6$ & 305.2 & -11.52 \\
\hline 93 & RXJ1902.7-3418 & $19: 02: 45.6$ & $-34: 18: 36.0$ & 8.8 & $\geq 0.47$ & $0.67 \pm 0.46$ & $5.3 \pm 3.3$ & 288.8 & -10.87 \\
\hline 94 & RXJ1903.0-3906 & 19:03:01.6 & $-39: 06: 09.4$ & 12.9 & $\geq 0.50$ & $0.20 \pm 0.37$ & $10.4 \pm 4.1$ & 222.5 & -10.87 \\
\hline 95 & $0-4009$ & 19:0 & $-40: 09: 09.7$ & 18.6 & $0.63 \pm 0.34$ & $0.03 \pm 0.34$ & $13.6 \pm 4.3$ & 194.3 & -10.93 \\
\hline 96 & RXJ1904.0-3817 & 19:04:01.7 & $-38: 17: 05.0$ & 7.7 & $\geq 0.28$ & $\leq-0.39$ & $4.4 \pm 2.8$ & 286.9 & -10.87 \\
\hline 97 & XJ1904.0-3804 & 19:04:04.0 & $-38: 04: 19.0$ & 10.2 & $-0.64 \pm 0.39$ & $-0.76 \pm 1.61$ & $13.5 \pm 4.8$ & 299.3 & -11.31 \\
\hline 98 & RXJ1904.0-3824 & 19:04:04.7 & $-38: 24: 56.8$ & 7.4 & $\leq-0.14$ & & $5.2 \pm 3.0$ & 293.0 & -11.52 \\
\hline 99 & RXJ1904.3-3835 & 19:04:23.7 & $-38: 35: 15.9$ & 10.6 & $\geq 0.42$ & $\geq 0.36$ & $7.4 \pm 3.8$ & 289.8 & -10.87 \\
\hline 100 & RXJ1904.5-3307 & 19:04:34.0 & $-33: 07: 16.0$ & 8.0 & $0.91 \pm 0.38$ & $0.27 \pm 0.39$ & $7.9 \pm 3.7$ & 115.6 & -10.88 \\
\hline 101 & RXJ1904.5-4028 & 19:04:34.4 & $-40: 28: 31.9$ & 7.5 & $\leq-0.29$ & $\leq 0.77$ & $7.4 \pm 3.8$ & 218.5 & -11.52 \\
\hline 102 & RXJ1904.6-4048 & 19:04:36.4 & $-40: 48: 24.2$ & 8.9 & $\geq 0.42$ & $-0.15 \pm 0.44$ & $7.3 \pm 3.4$ & 211.3 & -10.87 \\
\hline 103 & RXJ1904.7-3604 & 19:04:43.8 & $-36: 04: 58.5$ & 17.8 & $0.60 \pm 0.26$ & $0.33 \pm 0.29$ & $17.6 \pm 5.1$ & 326.7 & -10.94 \\
\hline 104 & XJ1905.0-3817 & 19:05:04.9 & $-38: 17: 46.3$ & 9.6 & $0.09 \pm 0.36$ & $-0.04 \pm 0.49$ & $11.7 \pm 4.3$ & 313.2 & -11.06 \\
\hline 105 & RXJ1905.0-3629 & 19:05:05.2 & $-36: 29: 14.0$ & 8.8 & $0.86 \pm 0.74$ & $\leq-0.50$ & $6.8 \pm 3.6$ & 332.1 & -10.89 \\
\hline 106 & RXJ1905.3-3855 & 19:05:18.8 & $-38: 55: 15.1$ & 12.1 & $0.70 \pm 0.33$ & $0.07 \pm 0.35$ & $17.5 \pm 5.1$ & 304.2 & -10.92 \\
\hline 107 & XJ1905.3-4030 & 19:05:19.6 & $-40: 30: 59.1$ & 8.5 & $-0.34 \pm 0.36$ & $-0.28 \pm 0.71$ & $12.3 \pm 4.5$ & 247.9 & -11.19 \\
\hline 108 & RXJ1906.4-3703 & 19:06:25.0 & $-37: 03: 34.9$ & 25.2 & $-0.80 \pm 0.25$ & $\geq-0.10$ & $14.3 \pm 4.2$ & 325.1 & -11.39 \\
\hline 109 & RXJ1906.5-3932 & $19: 06: 31.9$ & $-39: 32: 58.1$ & 8.8 & $-0.93 \pm 0.55$ & $\leq 0.06$ & $8.6 \pm 3.9$ & 289.6 & -11.47 \\
\hline
\end{tabular}


Table 2. continued

\begin{tabular}{|c|c|c|c|c|c|c|c|c|c|}
\hline No. & Designation & $\alpha_{2000}(\mathrm{X}$ & -ray) $\delta_{2000}$ & $M L$ & $H R 1$ & $H R 2$ & Counts & Exp. [s] & $\overline{\log E C F}$ \\
\hline 110 & RXJ1906.8-3748 & $19: 06: 51.2$ & $-37: 48: 40.3$ & 12.8 & $\leq-0.55$ & & $8.4 \pm 3.4$ & 325.3 & -11.52 \\
\hline 111 & RXJ1907.8-3923 & 19:07:50.8 & $-39: 23: 39.8$ & 74.9 & $\geq 0.82$ & $0.33 \pm 0.18$ & $28.8 \pm 5.9$ & 294.9 & -10.87 \\
\hline 112 & RXJ1909.6-3949 & 19:09:40.9 & $-39: 49: 37.6$ & 35.1 & $0.52 \pm 0.22$ & $0.26 \pm 0.26$ & $20.4 \pm 5.0$ & 322.2 & -10.96 \\
\hline 113 & RXJ1909.8-3343 & 19:09:50.9 & $-33: 43: 17.9$ & 10.6 & $\leq-0.31$ & & $7.0 \pm 3.4$ & 222.6 & -11.52 \\
\hline 114 & RXJ1910.8-3854 & 19:10:48.8 & $-38: 54: 48.4$ & 20.0 & $-0.04 \pm 0.27$ & $-0.42 \pm 0.36$ & $20.7 \pm 5.4$ & 351.6 & -11.09 \\
\hline 115 & RXJ1911.5-3434 & $19: 11: 34.8$ & $-34: 34: 59.3$ & 40.6 & $0.68 \pm 0.17$ & $0.13 \pm 0.24$ & $26.9 \pm 6.0$ & 323.7 & -10.92 \\
\hline 116 & RXJ1911.7-3641 & $19: 11: 47.4$ & $-36: 41: 54.9$ & 11.3 & $-0.76 \pm 0.41$ & $0.81 \pm 1.14$ & $10.3 \pm 3.9$ & 320.2 & -11.37 \\
\hline 117 & XJ1912.6-3431 & 19:12:36.6 & $-34: 31: 40.5$ & 16.7 & $0.57 \pm 0.34$ & $0.09 \pm 0.36$ & $13.8 \pm 4.5$ & 322.2 & -10.95 \\
\hline 118 & RXJ1913.0-3633 & 19:13:01.2 & $-36: 33: 04.4$ & 22.2 & $0.68 \pm 0.24$ & $0.00 \pm 0.30$ & $16.4 \pm 4.7$ & 319.0 & -10.92 \\
\hline 119 & RXJ1913.1-3622 & $19: 13: 10.2$ & $-36: 22: 06.1$ & 8.6 & $\geq 0.39$ & $-0.59 \pm 0.38$ & $7.6 \pm 3.5$ & 318.8 & -10.87 \\
\hline 120 & RXJ1913.3-3530 & $19: 13: 21.8$ & $-35: 30: 40.1$ & 8.3 & $\geq 0.27$ & $\leq-0.35$ & $4.7 \pm 2.8$ & 317.9 & -10.87 \\
\hline 121 & XJ1913.6-3543 & $19: 1$ & $-35: 43: 10.1$ & 11.7 & $\geq 0.54$ & $\leq-0.39$ & $5.8 \pm 3.3$ & 307.7 & -10.87 \\
\hline 122 & 2XJ1913.7-3303 & 19:13:46.3 & $-33: 03: 59.8$ & 9.3 & $0.51 \pm 0.32$ & $-0.15 \pm 0.42$ & $10.8 \pm 4.1$ & 194.9 & -10.96 \\
\hline 123 & RXJ1913.8-3347 & $19: 13: 50.2$ & $-33: 47: 49.1$ & 13.9 & $-0.06 \pm 0.38$ & $0.36 \pm 0.55$ & $15.8 \pm 5.2$ & 275.4 & -11.10 \\
\hline 124 & RXJ1914.4-3532 & 19:14:28.9 & $-35: 32: 27.2$ & 8.1 & $\geq 0.14$ & $\leq-0.19$ & $7.5 \pm 3.6$ & 292.3 & -10.87 \\
\hline 125 & RXJ191 & $19: 1$ & $-36: 01: 12.9$ & 8.0 & $\leq-0.37$ & & $8.4 \pm 4.0$ & 286.1 & -11.52 \\
\hline 126 & 759 & $19: 1$ & $-37: 59: 35.3$ & 8.5 & $\leq-0.49$ & & $4.6 \pm 2.7$ & 316.6 & -11.52 \\
\hline 127 & RXJ1915.5-3528 & $19: 15: 34.3$ & $-35: 28: 45.2$ & 7.8 & $0.69 \pm 0.43$ & \pm 0.36 & $9.8 \pm 4.2$ & 283.4 & -10.92 \\
\hline 128 & RXJ1915.7-3321 & $19: 15: 46.7$ & $-33: 21: 58.7$ & 9.7 & $\geq 0.37$ & $.59 \pm 0.37$ & $5.7 \pm 2.9$ & 228.0 & -10.87 \\
\hline 129 & 350 & $19: 1$ & $-33: 50: 47.7$ & 9.3 & $\geq 0.31$ & $\leq-0.50$ & $5.1 \pm 3.2$ & 267.5 & -10.87 \\
\hline 130 & 548 & $19: 1$ & $-35: 48: 20.1$ & 11.3 & $.57 \pm 0.25$ & $35 \pm 0.37$ & $13.6 \pm 4.6$ & 83.9 & -10.94 \\
\hline 131 & 3XJ1916 & 19:16:33.7 & $-34: 58: 55.7$ & 9.2 & $\leq-0.35$ & $\leq 0.47$ & $9.2 \pm 4.1$ & 292.7 & -11.52 \\
\hline 132 & RXJ1916.9-4024 & $19: 16: 58.2$ & $-40: 24: 32.0$ & 7.8 & $85 \pm 0.45$ & $\geq-0.50$ & $6.0 \pm 2.9$ & 246.5 & -11.42 \\
\hline 133 & RXJ1917.0-3331 & 19:17:04.0 & $-33: 31: 04.4$ & 28.0 & $\geq 0.63$ & $0.77 \pm 0.19$ & $17.0 \pm 5.0$ & 219.5 & -10.87 \\
\hline 134 & 31 & 19: & $-39: 31: 25.8$ & 7.6 & $\geq 0.20$ & $\leq-0.06$ & $4.1 \pm 2.5$ & 7.9 & -10.87 \\
\hline 135 & 756 & $19: 1$ & $-37: 56: 55.4$ & 122.6 & $0.46 \pm 0.12$ & $0.08 \pm 0.16$ & $58.1 \pm 8.2$ & 10.7 & -10.97 \\
\hline 136 & XJ1917.8-3927 & $19: 17$ & $-39: 27: 33.4$ & 7.8 & $\geq 0.41$ & $6 \pm 0.47$ & $5.7 \pm 3.1$ & 286.2 & -10.87 \\
\hline 137 & RXJ1918.0-4000 & 19:18:00.7 & $-40: 00: 28.6$ & 7.6 & $0.25 \pm 0.46$ & $\geq 0.24$ & $6.7 \pm 3.2$ & 250.7 & -11.02 \\
\hline 138 & RXJ19 & $19: 1$ & $-38: 23: 05.8$ & 44.6 & $-0.08 \pm 0.06$ & $20 \pm 0.08$ & $19.8 \pm 18$ & 307.7 & -11.10 \\
\hline 139 & 13 & 19: & $-39: 13: 30.9$ & 7.7 & $0.62 \pm 0.37$ & $\leq-0.48$ & $7.8 \pm 3.6$ & 299.9 & -10.94 \\
\hline 140 & RXJ191! & $19: 1$ & $-40: 31: 34.7$ & 9.0 & $-0.02 \pm 0.40$ & $0.51 \pm 0.56$ & $12.1 \pm 4.4$ & 209.3 & -11.09 \\
\hline 141 & RXJ1919.5-3639 & $19: 19$ & $-36: 39: 37.2$ & 9.4 & $-0.96 \pm 0.51$ & $\leq 0.61$ & $8.3 \pm 3.9$ & 298.4 & -11.49 \\
\hline 142 & RXJ1919.9-3924 & 19:19:55.3 & $-39: 24: 56.6$ & 16.5 & $-0.40 \pm 0.31$ & $\geq 0.17$ & $14.8 \pm 4.7$ & 306.5 & -11.21 \\
\hline 143 & 4009 & 19: & $-40: 09: 22.0$ & 7.5 & $-0.52 \pm 0.36$ & $-0.43 \pm 0.82$ & $10.4 \pm 4.1$ & 252.7 & -11.25 \\
\hline 144 & RXJ & $19: 2$ & $-39: 11: 29.6$ & 7.9 & $0.66 \pm 0.36$ & $0.35 \pm 0.45$ & $7.3 \pm 3.3$ & 314.8 & -10.93 \\
\hline 145 & RXJ1920.9-3320 & $19: 20$ & $-33: 20: 06.7$ & 9.5 & $0.32 \pm 0.37$ & $0.12 \pm 0.45$ & $10.4 \pm 4.0$ & 257.8 & -11.00 \\
\hline 146 & RXJ1921.1-4002 & 19:21:09.2 & $-40: 02: 14.4$ & 9.6 & $\leq-0.55$ & & $8.6 \pm 4.1$ & 264.9 & -11.52 \\
\hline 147 & RXJ1921.4-3459 & $19: 2$ & $-34: 59: 04.4$ & 72.1 & $0.10 \pm 0.07$ & $4 \pm 0.11$ & $202.2 \pm 14.9$ & 300.4 & -11.11 \\
\hline 148 & RXJ1 & 19: & $-35: 49: 16.1$ & 9.8 & $\geq 0.54$ & $-0.72 \pm 0.33$ & $7.8 \pm 4.0$ & 311.7 & -10.87 \\
\hline 149 & RXJ1922.4-3806 & $19: 22: 24.3$ & $-38: 06: 13.6$ & 8.3 & $\geq 0.60$ & $-0.06 \pm 0.45$ & $6.7 \pm 3.4$ & 303.9 & -10.87 \\
\hline 150 & RXJ1922.9-3545 & $19: 22: 55.3$ & $-35: 45: 39.2$ & 21.4 & $\geq 0.68$ & $\geq 0.53$ & $10.5 \pm 3.9$ & 303.9 & -10.87 \\
\hline 151 & RXJ1923.3-3658 & $19: 23: 18.9$ & $-36: 58: 39.3$ & 9.7 & $-0.05 \pm 0.37$ & $0 \pm 0.56$ & $10.2 \pm 4.0$ & 320.7 & -11.10 \\
\hline 152 & $\mathrm{RX}$ & $19: 2$ & $-35: 5$ & 8.7 & $\geq 0.41$ & $0.48 \pm 0.47$ & $6.2 \pm 3.2$ & 298.5 & -10.87 \\
\hline 153 & RXJ1923.8-3700 & $19: 23: 51.9$ & $-37: 00: 08.2$ & 7.6 & $0.51 \pm 0.34$ & $-0.02 \pm 0.51$ & $7.4 \pm 3.3$ & 311.9 & -10.96 \\
\hline 154 & RXJ1923.8-4036 & $19: 23: 53.3$ & $-40: 36: 46.4$ & 66.9 & $-0.08 \pm 0.19$ & $-0.35 \pm 0.27$ & $33.1 \pm 6.3$ & 275.6 & -11.10 \\
\hline 155 & RXJ1924.1-3333 & 19:24:09.9 & $-33: 33: 10.2$ & 8.0 & $0.74 \pm 0.40$ & $0.32 \pm 0.42$ & $9.7 \pm 4.2$ & 296.8 & -10.91 \\
\hline 156 & RXJ1924.3-4008 & $19: 24: 20.2$ & $-40: 08: 35.5$ & 7.7 & $0.02 \pm 0.46$ & $\leq-0.11$ & $10.0 \pm 4.3$ & 309.0 & -11.08 \\
\hline 157 & RXJ1924.4-3959 & $19: 24: 27.9$ & $-39: 59: 29.1$ & 10.3 & $0.53 \pm 0.38$ & $0.8 \overline{7} \pm 0.32$ & $6.9 \pm 3.2$ & 307.9 & -10.95 \\
\hline 158 & RXJ1924.5-3442 & $19: 24: 34.0$ & $-34: 42: 34.6$ & 20.5 & $-0.29 \pm 0.31$ & $0.95 \pm 0.47$ & $15.0 \pm 4.5$ & 298.9 & -11.17 \\
\hline 159 & RXJ1924.7-3601 & $19: 24: 47.3$ & $-36: 01: 15.1$ & 7.5 & $-0.09 \pm 0.45$ & $0.20 \pm 0.77$ & $7.7 \pm 3.5$ & 297.7 & -11.11 \\
\hline 160 & RXJ1925.0-3621 & $19: 25: 02.7$ & $-36: 21: 39.1$ & 10.1 & $0.07 \pm 0.40$ & $-0.22 \pm 0.54$ & $8.2 \pm 3.4$ & 297.3 & -11.06 \\
\hline 161 & RXJ1925.0-3549 & $19: 25: 03.9$ & $-35: 49: 34.4$ & 7.6 & $\geq 0.39$ & $0.60 \pm 0.32$ & $10.8 \pm 4.6$ & 297.0 & -10.87 \\
\hline 162 & RXJ1925.3-3413 & $19: 25: 21.4$ & $-34: 13: 06.4$ & 7.4 & $\leq-0.49$ & $\leq 0.88$ & $9.6 \pm 4.3$ & 312.5 & -11.52 \\
\hline 163 & RXJ1926.5-3426 & $19: 26: 31.8$ & $-34: 26: 25.7$ & 7.6 & $\leq-0.47$ & $\geq-0.60$ & $6.8 \pm 3.5$ & 300.9 & -11.52 \\
\hline 164 & RXJ1927.1-3713 & $19: 27: 06.2$ & $-37: 13: 42.2$ & 14.6 & $\geq 0.65$ & $0.7 \overline{7} \pm 0.30$ & $10.2 \pm 4.0$ & 316.9 & -10.87 \\
\hline 165 & RXJ1927.4-3847 & $19: 27: 28.0$ & $-38: 47: 06.9$ & 28.3 & $\geq 0.71$ & $0.29 \pm 0.25$ & $17.3 \pm 5.0$ & 307.7 & -10.87 \\
\hline 166 & RXJ1927.9-3955 & $19: 27: 55.0$ & $-39: 55: 03.8$ & 7.9 & $\leq-0.44$ & $\leq 0.98$ & $5.5 \pm 3.0$ & 325.6 & -11.52 \\
\hline
\end{tabular}


Table 2. continued

\begin{tabular}{|c|c|c|c|c|c|c|c|c|c|}
\hline No. & Designation & $\alpha_{2000}(\mathrm{X}$ & (-ray) $\delta_{2000}$ & $M L$ & $H R 1$ & $H R 2$ & Counts & Exp. $[\mathrm{s}]$ & $\log E C F$ \\
\hline 167 & RXJ1928.0-4050 & $19: 28: 04.8$ & $-40: 50: 04.0$ & 46.2 & $0 \pm 0.17$ & $00 \pm 0.22$ & $31.1 \pm 6.5$ & 322.3 & -10.94 \\
\hline 168 & RXJ & 3.1 & $-33: 52$ & 11.9 & $\geq 0.45$ & $03 \pm 0.45$ & $8.2 \pm 3.5$ & 1.6 & -10.87 \\
\hline 169 & XJ1928.2-3915 & 13.3 & $-39: 15: 13.5$ & 7.4 & $.76 \pm 0.70$ & $\leq-0.33$ & $5.1 \pm 3.0$ & 20.8 & -10.91 \\
\hline 170 & RXJ1928.3-4031 & $19: 28: 20.6$ & $-40: 31: 19.7$ & 10.3 & $\geq 0.48$ & $0.85 \pm 0.54$ & $9.4 \pm 4.4$ & 325.7 & -10.87 \\
\hline 171 & RXJ1928.5-3508 & $19: 28: 31.6$ & $-35: 08: 02.0$ & 268.9 & $0.83 \pm 0.07$ & $0.27 \pm 0.11$ & $88.8 \pm 9.9$ & 307.2 & -10.90 \\
\hline 172 & 646 & $19: 2$ & $-36: 46: 38.6$ & 13.6 & $\geq 0.52$ & $0.15 \pm 0.35$ & $9.7 \pm 3.7$ & 08.9 & -10.87 \\
\hline 173 & 3805 & 3.2 & $-38: 05: 15.8$ & 8.6 & $39 \pm 0.57$ & $\geq 0.42$ & $7.5 \pm 4.0$ & 24.6 & -10.98 \\
\hline 174 & RXJ1929.2-4055 & 19:29:16.0 & $-40: 55: 20.4$ & 9.1 & $\leq-0.43$ & & $5.4 \pm 2.8$ & 329.9 & -11.52 \\
\hline 175 & RXJ1929.6-4018 & $19: 29$ & $-40: 18: 53.6$ & 39.3 & $.80 \pm 0.17$ & $0.07 \pm 0.23$ & $26.9 \pm 5.9$ & 344.0 & -10.90 \\
\hline 176 & XJ19 & $19: 2$ & $-37: 02: 35.7$ & 14.8 & $.74 \pm 0.35$ & $\geq-0.03$ & $12.9 \pm 4.6$ & 318.8 & -11.36 \\
\hline 177 & 38 & .4 & $-33: 3$ & 8.9 & $\geq 0.38$ & $21 \pm 0.43$ & $8.5 \pm 3.9$ & 31.0 & -10.87 \\
\hline 178 & 302 & 3.1 & $-33: 02$ & 7.9 & $\leq-0.57$ & & $6.0 \pm 3.7$ & 48.9 & -11.52 \\
\hline 179 & XJ1930.6 & 39.2 & $-35: 25: 47.5$ & 13.8 & $\geq 0.61$ & \pm 0.31 & $13.6 \pm 4.7$ & 324.4 & -10.87 \\
\hline 180 & 441 & 8.1 & $-34: 41: 06.6$ & 11.0 & $\geq 0.39$ & $\leq-0.47$ & $9.6 \pm 4.1$ & 319.5 & -10.87 \\
\hline 18 & 08 & .1 & -40 & 8 & $0.65 \pm 0.39$ & $0.72 \pm 0.38$ & $6.4 \pm 3.2$ & 4.1 & -10.93 \\
\hline 182 & 40 & .5 & $-33: 4$ & 7.8 & $\leq-0.51$ & & $5.8 \pm 3.3$ & 72.9 & -11.52 \\
\hline 183 & .6-3354 & $19: 3$ & $-33: 54: 38.7$ & 96.7 & $.93 \pm 0.07$ & \pm 0.10 & $52.5 \pm 8.4$ & 285.2 & -10.88 \\
\hline 184 & XJ1932.3-3804 & $19: 3$ & $-38: 04: 58.6$ & 7.4 & $\geq 0.41$ & $0.67 \pm 0.32$ & $6.0 \pm 3.3$ & 324.9 & -10.87 \\
\hline 185 & 06 & 1 & $-35:$ & 7.8 & 0.47 & $-0.46 \pm 0.40$ & $8.0 \pm 3.8$ & 3.0 & -10.87 \\
\hline 186 & 37 & .1 & -38 & 10.6 & $\geq 0.22$ & $\leq-0.54$ & $6.5 \pm 3.6$ & 329.6 & -10.87 \\
\hline 187 & 32 & 19 & $-34: 32: 22.7$ & 11.8 & $.34 \pm 0.31$ & $0.50 \pm 0.63$ & $13.4 \pm 4.6$ & 305.7 & -11.19 \\
\hline 188 & 58 & 19 & $-38: 5$ & 9.5 & $8 \pm 0.54$ & 0.45 & $7.4 \pm 3.6$ & 336.0 & -10.89 \\
\hline 189 & $.9-3554$ & $19: 3$ & $-35: 54: 23.0$ & 7.9 & $\geq 0.30$ & $\leq-0.13$ & $5.6 \pm 3.2$ & 314.9 & -10.87 \\
\hline 190 & 37 & 3.1 & $: 29.4$ & 10.5 & $5 \pm 0.50$ & $\geq 0.26$ & $8.8 \pm 3.9$ & 284.4 & -10.95 \\
\hline 191 & 12 & .2 & -38 & 14.7 & $0.68 \pm 0.40$ & $-0.08 \pm 0.39$ & $10.9 \pm 4.0$ & 350.1 & -10.92 \\
\hline 192 & 743 & $19: 3$ & $-37: 43: 46.7$ & 7.4 & $\leq-0.58$ & & $5.6 \pm 3.5$ & 336.1 & -11.52 \\
\hline 193 & $.6-3453$ & 19:33:41.6 & $-34: 53: 27.1$ & 11.8 & $0.77 \pm 0.34$ & $48 \pm 0.37$ & $13.2 \pm 4.7$ & 321.7 & -10.91 \\
\hline 194 & 22 & .3 & -3 & 7.6 & $0.29 \pm 0.47$ & $\leq-0.03$ & $9.0 \pm 3.9$ & 320.6 & -11.17 \\
\hline 195 & 20 & 3.6 & -36 & 9.0 & $\geq 0.51$ & $0.06 \pm 0.42$ & $6.8 \pm 3.7$ & 318.2 & -10.87 \\
\hline 196 & XJ1934.6-3433 & $19: 3$ & $-34: 33: 33.2$ & 7.7 & $\leq-0.40$ & & $9.0 \pm 4.2$ & 328.1 & -11.52 \\
\hline 197 & XJ1934.7-3805 & $19: 3$ & $-38: 05: 06.0$ & 52.9 & $0.76 \pm 0.18$ & $6 \pm 0.20$ & $30.8 \pm 6.3$ & 342.6 & -10.91 \\
\hline 198 & & 19 & $-35: 36: 10.9$ & 9. & $0.42 \pm 0.47$ & $0.87 \pm 0.57$ & $8.3 \pm 3.5$ & 329.8 & -10.98 \\
\hline 199 & 25 & $19::$ & $-33: 2$ & 8.8 & $0.89 \pm 0.38$ & $\geq 0.16$ & $4.5 \pm 2.7$ & 274.4 & -10.88 \\
\hline 200 & XJ1936.0-4002 & $19: 3$ & $-40: 02: 24.6$ & 10.7 & $-0.21 \pm 0.39$ & $\geq 0.24$ & $9.9 \pm 3.8$ & 335.3 & -11.14 \\
\hline 201 & XJ1936.9-3429 & $19: 36: 56.9$ & $-34: 29: 28.0$ & 9.4 & $0.28 \pm 0.42$ & $\leq-0.44$ & $8.7 \pm 3.8$ & 305.6 & -11.01 \\
\hline 202 & XJ1937.2-3958 & $19: 37: 12.6$ & $-39: 58: 01.1$ & 22.1 & $0.41 \pm 0.26$ & $0.21 \pm 0.29$ & $21.6 \pm 5.6$ & 333.6 & -10.98 \\
\hline 203 & 10 & $19: 3$ & $-40: 10: 21.5$ & 11.8 & & $08 \pm 0.44$ & $8.8 \pm 4.0$ & 335.8 & -10.87 \\
\hline 204 & XXJ1938.0-3801 & 19:38:03.0 & $-38: 01: 05.8$ & 10.3 & $0.04 \pm 0.49$ & $\geq 0.46$ & $7.7 \pm 3.6$ & 346.1 & -11.07 \\
\hline 205 & XJ1938.1-3914 & 19:38:09.4 & $-39: 14: 02.4$ & 9.3 & $\geq 0.36$ & $\geq 0.42$ & $3.6 \pm 2.6$ & 345.0 & -10.87 \\
\hline 206 & RXJ1938.2-4006 & 19:38:13.1 & $-40: 06: 38.1$ & 7.8 & $0.55 \pm 0.40$ & $\geq 0.50$ & $7.6 \pm 4.0$ & 333.7 & -10.95 \\
\hline
\end{tabular}

$V$ magnitude, $\mathrm{X}$-ray to optical flux ratio, as well as some more information on the identified counterparts. See Sect. 3 for details on the identification. For calculating the $\mathrm{X}$-ray to optical flux ratio, we use

$f_{V}=10^{-0.4 \cdot(V+13.42)} \mathrm{erg} \mathrm{cm}^{-2} \mathrm{~s}^{-1}$

(with $V$ in mag) and the X-ray flux, which we get from the data in Table 2 via

$f_{\mathrm{X}}=E C F \cdot$ counts/exposure.

For sources where we can list only an upper or lower limit to HR 1 (in Table 2), we use $H R 1=1$ or $H R 1=-1$, respectively, for calculating the flux according to Eq. (2). The X-ray to optical flux ratio already gives some hints on the nature of an unidentified X-ray source: Extragalactic $\mathrm{X}$-ray sources are optically faint but X-ray bright with $\log \left(f_{\mathrm{X}} / f_{V}\right) \geq-0.5$, while normal stars usually have $\log \left(f_{\mathrm{X}} / f_{V}\right) \leq-1$ (Stocke et al. 1983; Motch et al. 1998).

\section{Optical follow-up spectroscopy}

Out of the $206 \mathrm{X}$-ray sources, 89 have one or several nearby ( $\leq 40$ arcsec) potential optical counterparts (brighter than $V=16 \mathrm{mag}$ ) in Simbad and/or GSC, including four previously known PMS stars (namely CrAPMS 1, 2, 3, and TY CrA) and four previously known older stars. In addition, there is one optically faint neutron star $(V=$ $25.7 \mathrm{mag}$, see footnote 2) and three extra-galactic sources (see Table 3). To identify a large fraction of the remaining 81 unidentified X-ray sources, we performed low-resolution 
Table 3. Optical counterparts. For each X-ray source listed in Table 2, we list here the optical counterpart from Simbad, GSC, or NED, unless no counterpart is found within 40 arcsec around the X-ray position (in all but four cases, these counterparts are the closest ones). We list running number as in Table 2, optical position, offset between X-ray and optical position (in arcsec), optical magnitude ( $V$ in mag), and the (log of the) X-ray to optical flux ratio. In the Col. ID, we list the nature of the counterpart, i.e. $\mathrm{y}$ for new young star listed in Table 4, z for zero-age main-sequence stars (i.e. low lithium), $\mathrm{d}$ for $\mathbf{d K e}$ or $\mathbf{d M e}$ stars, e for extra-galactic, n for neither of the above (mostly old stars), p for previously known TTS or non-TTS (not observed optically except TY CrA); counterparts without any entry in the Col. ID have not been observed optically. Finally, some remarks are given as found in Simbad (like proper motion PM as $\left[\mu_{\alpha} \cdot \cos \delta, \mu_{\delta}\right]$ in milli arc seconds per year and radial velocity $R V$ in $\mathrm{km} \mathrm{s}^{-1}$ ). or from our optical follow-up observations. Data on optical counterparts are taken from Simbad or NED, if remarks are listed, otherwise from GSC. Some GSC counterparts appear on several GSC plates which may have different colors and different filters; we have always chosen the closest counterpart. For stars with $V$ mag given with colons, positions and magnitudes are estimated from the DSS charts, because the identified counterpart is different from the relevant Simbad/GSC/NED counterpart

\begin{tabular}{|c|c|c|c|c|c|c|c|}
\hline \multirow{2}{*}{$\begin{array}{r}\text { No. } \\
\text { Table } 2\end{array}$} & \multicolumn{2}{|c|}{ Optical position } & \multirow{2}{*}{$\begin{array}{c}\Delta \\
{\left[{ }^{\prime \prime}\right]}\end{array}$} & \multirow{2}{*}{$\begin{array}{r}V \\
\text { mag }\end{array}$} & \multirow{2}{*}{$\begin{array}{c}\log \\
f_{\mathrm{X}} / f_{V}\end{array}$} & \multirow[t]{3}{*}{ ID } & \multirow[t]{2}{*}{ Remarks } \\
\hline & $\alpha_{2000}$ & $\delta_{2000}$ & & & & & \\
\hline 1 & $18: 35: 06.2$ & $-34: 04: 02.8$ & 28 & 13.9 & -1.15 & & \\
\hline 3 & $18: 35: 46.6$ & $-32: 59: 31.2$ & 12 & 9.8 & -1.24 & $\mathrm{p}$ & globular cluster NGC 6652 \\
\hline 8 & $18: 36: 39.5$ & $-34: 51: 25.0$ & 16 & 13.0 & -1.45 & $\mathrm{y}$ & \\
\hline 9 & $18: 37: 17.6$ & $-34: 42: 42.2$ & 8 & 11.3 & -2.31 & $\mathrm{n}$ & \\
\hline 11 & $18: 38: 20.2$ & $-35: 23: 37.2$ & 5 & 12.2 & -2.26 & $\mathrm{~d}$ & dKe star \\
\hline 12 & $18: 39: 04.9$ & $-37: 05: 27.8$ & 22 & 13.1 & -1.30 & $\mathrm{n}$ & \\
\hline 13 & $18: 39: 05.3$ & $-37: 26: 21.8$ & 16 & 10.9 & -2.35 & $\mathrm{y}$ & \\
\hline 17 & $18: 40: 37.6$ & $-37: 28: 18.1$ & 28 & 11.2 & -2.27 & $\mathrm{~d}$ & dKe star \\
\hline 19 & $18: 40: 53.3$ & $-35: 46: 44.6$ & 22 & 14.5: & -0.84 & $\mathrm{y}$ & \\
\hline 23 & $18: 41: 48.6$ & $-35: 25: 43.6$ & 14 & 9.7 & -2.44 & $\mathrm{y}$ & \\
\hline 27 & $18: 42: 58.0$ & $-35: 32: 42.9$ & 10 & 12.2 & -1.73 & $\mathrm{y}$ & \\
\hline 30 & $18: 44: 21.9$ & $-35: 41: 43.6$ & 29 & 11.3 & -1.81 & $\mathrm{y}$ & \\
\hline 31 & $18: 44: 31.1$ & $-37: 23: 34.3$ & 26 & 13.1 & -1.13 & $\mathrm{y}$ & \\
\hline 35 & $18: 45: 09.3$ & $-33: 24: 03.9$ & 26 & 12.3 & -2.18 & $\mathrm{n}$ & \\
\hline 36 & $18: 45: 34.8$ & $-37: 50: 19.6$ & 7 & 9.2 & -2.45 & $\mathrm{y}$ & HD 173148, G5 V, $P M=[4.7,-32.0]$ \\
\hline 38 & $18: 46: 43.9$ & $-36: 04: 52.2$ & 13 & 12.0 & -2.14 & & IRAS $18433-3608$ \\
\hline 39 & $18: 46: 45.6$ & $-36: 36: 18.1$ & 8 & 10.3 & -2.45 & $\mathrm{y}$ & \\
\hline 42 & $18: 47: 14.0$ & $-37: 09: 48.3$ & 6 & 12.1 & -2.09 & & \\
\hline 45 & $18: 47: 44.6$ & $-40: 24: 22.2$ & 34 & 5.2 & -4.18 & $\mathrm{n}$ & $\mu \mathrm{CrA}, \mathrm{G} 5.5 \mathrm{I}, P M=[24.6,-18.6], R V=-18.2, d=120 \mathrm{pc}$ \\
\hline 48 & $18: 48: 35.8$ & $-34: 58: 20.4$ & 38 & 13.3 & -1.73 & & \\
\hline 53 & $18: 52: 17.3$ & $-37: 00: 12.0$ & 14 & 13.9 & -0.98 & $\mathrm{y}$ & IRAS $18489-3703$ \\
\hline 54 & $18: 52: 24.8$ & $-37: 30: 35.6$ & 10 & 12.5 & -1.57 & $\mathrm{~d}$ & dMe star \\
\hline 55 & $18: 53: 06.0$ & $-36: 10: 22.8$ & 28 & 9.6 & -2.47 & $\mathrm{y}$ & HD 174656, G6 IV, $P M=[-1.2,-34.0]$ \\
\hline 59 & $18: 54: 29.0$ & $-37: 39: 04.5$ & 26 & 11.8 & -2.23 & & \\
\hline 63 & $18: 56: 37.3$ & $-37: 54: 26.9$ & 26 & 25.7 & +4.77 & $\mathrm{p}$ & neutron star RXJ1856.5-3754 \\
\hline 64 & $18: 56: 44.0$ & $-35: 45: 31.9$ & 10 & 13.0 & -2.08 & $\mathrm{y}$ & \\
\hline 65 & $18: 56: 49.2$ & $-40: 21: 07.0$ & 34 & 14.9 & -0.92 & & \\
\hline 68 & $18: 57: 34.1$ & $-37: 32: 32.3$ & 25 & 15.0: & -1.02 & $\mathrm{y}$ & \\
\hline 73 & $18: 58: 43.4$ & $-37: 06: 26.5$ & 7 & 4.9 & -4.86 & $\mathrm{p}$ & $\epsilon \mathrm{CrA}, \mathrm{F} 2 \mathrm{~V}, \mathrm{~W}$ UMa-type, $d=30 \mathrm{pc}$ \\
\hline 77 & $19: 00: 49.5$ & $-34: 52: 49.2$ & 33 & 8.4 & -3.61 & & HD $176247, \mathrm{G} 1 \mathrm{~V}, P M=[23.4,-24.0]$ \\
\hline 80 & 19:01:09.5 & $-36: 47: 51.7$ & 11 & 12.7 & -1.97 & $\mathrm{y}$ & VSS VIII-27 \\
\hline 81 & 19:01:26.7 & $-40: 22: 34.0$ & 24 & 13.6 & -1.37 & $\mathrm{n}$ & \\
\hline 82 & 19:01:28.7 & $-34: 22: 35.5$ & 25 & 8.2 & -3.29 & $\mathrm{y}$ & HD 176383, F5 V, $P M=[9.9,-46.8]$ \\
\hline 83 & 19:01:34.9 & $-37: 00: 55.8$ & 7 & 11.3 & -2.03 & $\mathrm{p}$ & CrAPMS 1, K1 IV, wTTS \\
\hline 84 & 19:01:40.8 & $-36: 52: 34.2$ & 30 & 9.5 & -2.79 & $\mathrm{p}$ & TY CrA, Herbig Ae/Be PMS star \\
\hline 85 & 19:01:40.5 & $-36: 44: 31.9$ & 27 & 13.0: & -1.56 & $\mathrm{y}$ & VSS VIII-26 \\
\hline 87 & 19:02:01.9 & $-37: 07: 43.2$ & 14 & 10.4 & -2.57 & $\mathrm{p}$ & CrAPMS 2, G5 IV, wTTS, $P M=[0.0,-33.0]$ \\
\hline 89 & 19:02:22.1 & $-36: 55: 40.8$ & 2 & 13.8 & -1.60 & $\mathrm{p}$ & CrAPMS 3, K2 IV, wTTS \\
\hline 90 & 19:02:22.7 & $-39: 22: 21.9$ & 11 & 14.2 & -1.46 & & \\
\hline 91 & $19: 02: 25.9$ & $-36: 17: 39.0$ & 32 & & & $\mathrm{e}$ & galaxy CGMW 4-4634 \\
\hline 93 & $19: 02: 43.6$ & $-34: 19: 00.4$ & 35 & 13.7 & -1.75 & & \\
\hline 95 & 19:03:00.6 & $-40: 09: 16.8$ & 33 & 13.4 & -1.35 & $\mathrm{n}$ & \\
\hline 97 & $19: 03: 58.4$ & $-38: 04: 01.0$ & 70 & 13.1 & -2.04 & $\mathrm{z}$ & \\
\hline 102 & $19: 04: 38.8$ & $-40: 48: 15.4$ & 29 & 11.4 & -2.39 & & \\
\hline 108 & $19: 06: 24.8$ & $-37: 03: 41.7$ & 7 & 5.0 & -5.37 & $\mathrm{n}$ & $\gamma \mathrm{CrA}, \mathrm{F} 8 \mathrm{~V}, P M=[95,-274], R V=-51.0, d=21 \mathrm{pc}$ \\
\hline 110 & $19: 06: 52.5$ & $-37: 48: 37.6$ & 16 & 6.2 & -5.26 & $\mathrm{n}$ & HR $7232, \mathrm{G} 5 \mathrm{IV}, d=17 \mathrm{pc}$ \\
\hline 111 & 19:07:50.4 & $-39: 23: 32.2$ & 9 & 14.2 & -0.84 & $\mathrm{z}$ & \\
\hline
\end{tabular}


Table 3. continued

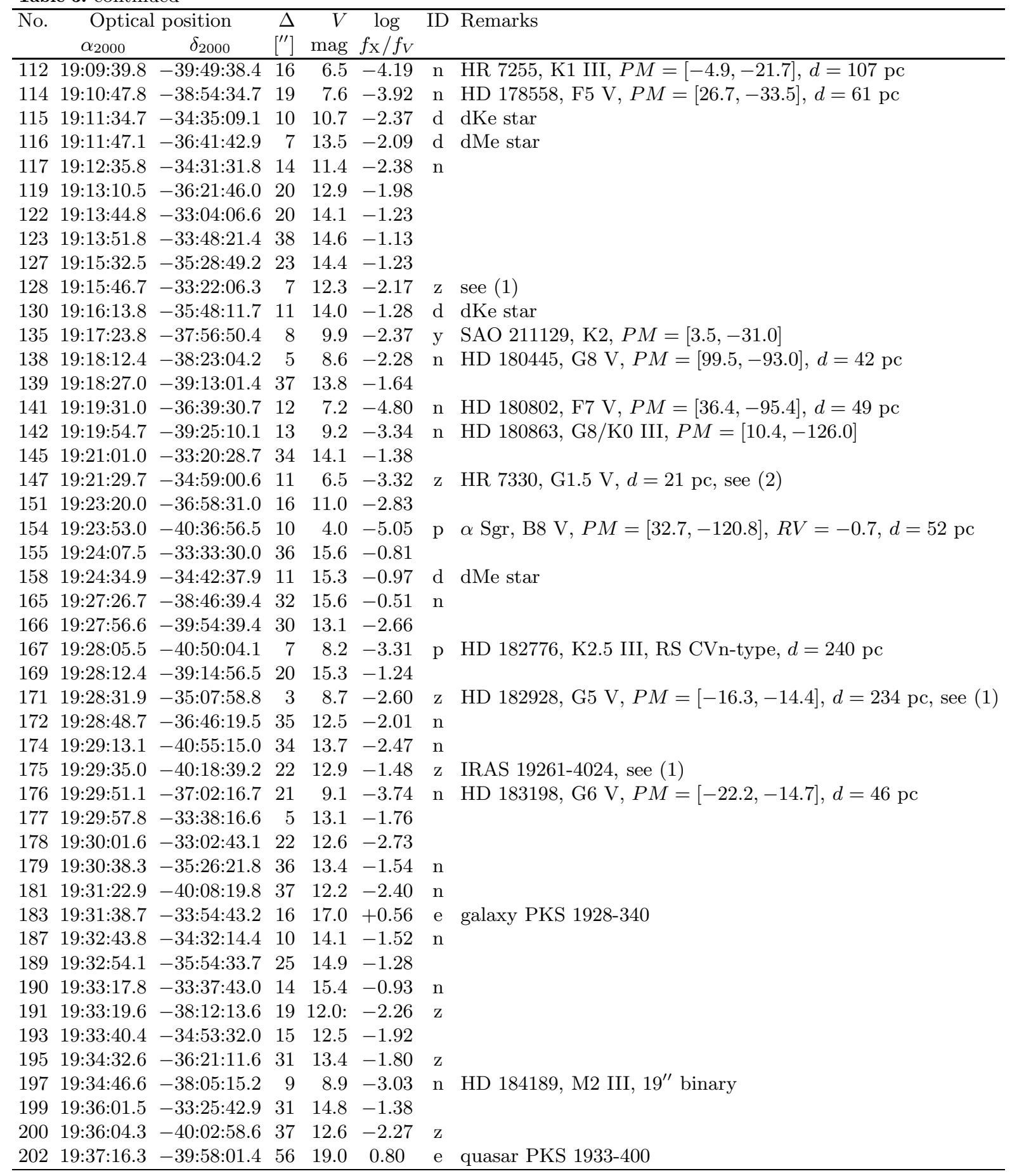

Notes: (1) Maybe weak lithium (or noise), complex H $\alpha$, maybe double-lined, could be ZAMS, RS CVn-type, dKe/dMe, or a cool Algol. (2) Our detection of weak lithium with $W\left(\lambda(\mathrm{Li})=0.19 \simeq W_{\lambda}(\mathrm{Ca})\right.$ confirms the classification of HR 7330 as member of the $\sim 200$ Myr old nearby Castor moving group by Barrado y Navascues (1998). 
spectroscopy of 148 stars, which are potential optical counterparts to 56 of those 81 X-ray sources.

Low-dispersion spectra were taken with the Boller \& Chivens spectrograph of the ESO $1.5 \mathrm{~m}$ telescope on La Silla in twelve nights, namely 1995 July $16 / 17$ to $21 / 22$ and 1996 July $20 / 21$ to $25 / 26$. The wavelength range is 4500 to $6850 \AA$ and the spectral resolution is $\sim 2.5 \AA$. For technical details and data reduction, we refer to the relevant part of Sect. 2.2 in Walter et al. (1997).

In addition to those potential RASS source counterparts, we also observed a number of previously known or suspected young stars in CrA, because their lithium line strength was not known, see Table 1 for those data; most of these spectra were taken with the Boller \& Chivens spectrograph. However, the stars HBC 673, 675, 677, and CrAPMS 3/c were observed with the ESO-3.5 m-NTT ${ }^{4}$. Here, we observed in the red medium-dispersion mode (EMMI red arm, CCD \# 36, grating 6) at a resolution of 5500 in the wavelength range from 6160 to $7740 \AA$. While we could not detect lithium in HBC 673 and 675 , we confirmed HBC 677 to be a cTTS and CrAPMS 3/c to be a wTTS (see Table 1 ).

For most of the RASS counterparts with detected lithium (in low-resolution spectra), we then took highresolution spectra to confirm their youth. The high dispersion spectra were obtained with the CTIO $4 \mathrm{~m}$ telescope and echelle spectrograph on 14 to 17 July 1998. We used the 226-3 cross disperser and the $31.6 \mathrm{l} / \mathrm{mm}$ echelle with the red optics. We used the SITe $2 \mathrm{~K} \# 6$ CCD detector, at a gain of 5 , corresponding to about $1 \mathrm{e}^{-}$per ADU and a read noise of about $3 \mathrm{e}^{-}$. We used the GG385 filter for order sorting. We used a $150 \mu \mathrm{m}$ (1 arcsec) slit and decker \#9 (3.3 arcsec) for the stellar observations. The seeing was generally 1 to 1.5 arcsec, and there were some clouds on 3 of the 4 nights. The spectra cover the range from roughly $4400 \AA$ through $7500 \AA$ at a resolution of 25000 . We obtained projector flat images to flatten the spectra. A Th-Ar comparison source was observed before and after each telescope slew. Each stellar observation was made in three parts to facilitate cosmic ray removal. Initial reductions were undertaken at CTIO, using the IRAF DOECSLIT package. We corrected for bias, extracted the orders, divided by the flats, and solved for the dispersion. The data were rebinned to a linear wavelength scale in each order. We removed the global background (the scattered light correction) but did not attempt to subtract the local background. The data were further reduced using IDL. We flattened the spectra in each order to remove any residual curvature left from the original flat division. We trimmed the ends of the orders. We then filtered the three individual spectra of each object to remove cosmic rays, and coadded the spectra. We determine

\footnotetext{
4 Taken during ESO program 63.L-0023 at the end of the night 18/19 April 1999, when the main targets of that run were not visible anymore.
}

radial velocities by cross-correlating the spectra against the sky spectra. We expect an internal precision of about $1 \mathrm{~km} \mathrm{~s}^{-1}$, except for the targets with poorer $S / N$.

\section{Results of the spectroscopy}

Stars which fullfill all the following conditions (according to their high-resolution spectra, if available, otherwise we use the low-resolution spectra) are classified as new PMS stars, i.e. appear as such in Table 4:

- Spectral type later than mid F, and

- lithium $6708 \AA$ line with $W_{\lambda}(\mathrm{Li}) \geq 0.1 \AA$, and

- more Li than ZAMS stars of the same spectral type, and

$-W_{\lambda}(\mathrm{Li}) \geq W_{\lambda}(\mathrm{Ca})$ (for spectral types $\mathrm{F}, \mathrm{G}$, and $\left.\mathrm{K}\right)^{5}$. All these stars are classified as young stars (letter $\mathbf{y}$ in Col. ID in Table 3).

Table 4 lists for all newly discovered TTS their official designation, PMS type (i.e. whether wTTS or cTTS), the spectral type, thje $\mathrm{H} \alpha$ and lithium equivalent widths, the projected rotational velocity, the radial velocity, the X-ray luminosity, and some remarks, e.g. on binarity.

All stars with a detected lithium line, which is weaker than $0.1 \AA$, and all F-, G-, and K-type stars with a detected lithium line, which is weaker than the $\mathrm{Ca}$ $6718 \AA$ line, are classified as ZAMS stars (letter $\mathbf{z}$ in Col. ID in Table 3). Stars without detectable lithium, but $\mathrm{H} \alpha$ emission are classified as $\mathbf{d K e}$ or dMe stars (letter $\mathbf{d}$ in Col. ID in Table 3).

In Table 3, we list the nature of the newly identified $\mathrm{X}$-ray sources. Among the $56 \mathrm{X}$-ray sources for which we performed low-resolution spectroscopy of potential optical counterparts, we identified 19 new pre-main sequence stars, nine new zero-age main-sequence stars, and seven new $\mathrm{dKe} / \mathrm{dMe}$ stars. Two of the new PMS stars have $W_{\lambda}(\mathrm{H} \alpha) \geq 10 \AA$, i.e. are classical TTS, and 17 are weak-line TTS, four of which form two visual pairs, which are spatially unresolved with ROSAT, i.e. one pair corresponds to one X-ray source each.

The spatial distribution of the CrA RASS sources and TTS is shown in Fig. 1. While all the previously known TTS in CrA including those found by EO cluster on the main dark cloud, the new ROSAT TTS are more widely distributed. In particular, they are almost all located west of the cloud. The two new cTTS appear to be situated in two small cloud-lets, where they most certainly have formed. The new wTTS may have also formed in such small cloud-lets outside the current borders of the CrA cloud, and those small cloud-lets may well have dispersed since they formed stars, similar to some seemingly offcloud wTTS near Cha I (Alcalá et al. 1995; Mizuno et al. 1998), see below.

\footnotetext{
${ }^{5}$ X-ray emitting M-type stars with lithium are always preMS stars, as they burn all Lithium before reaching the MS (see e.g. Covino et al. 1997).
} 


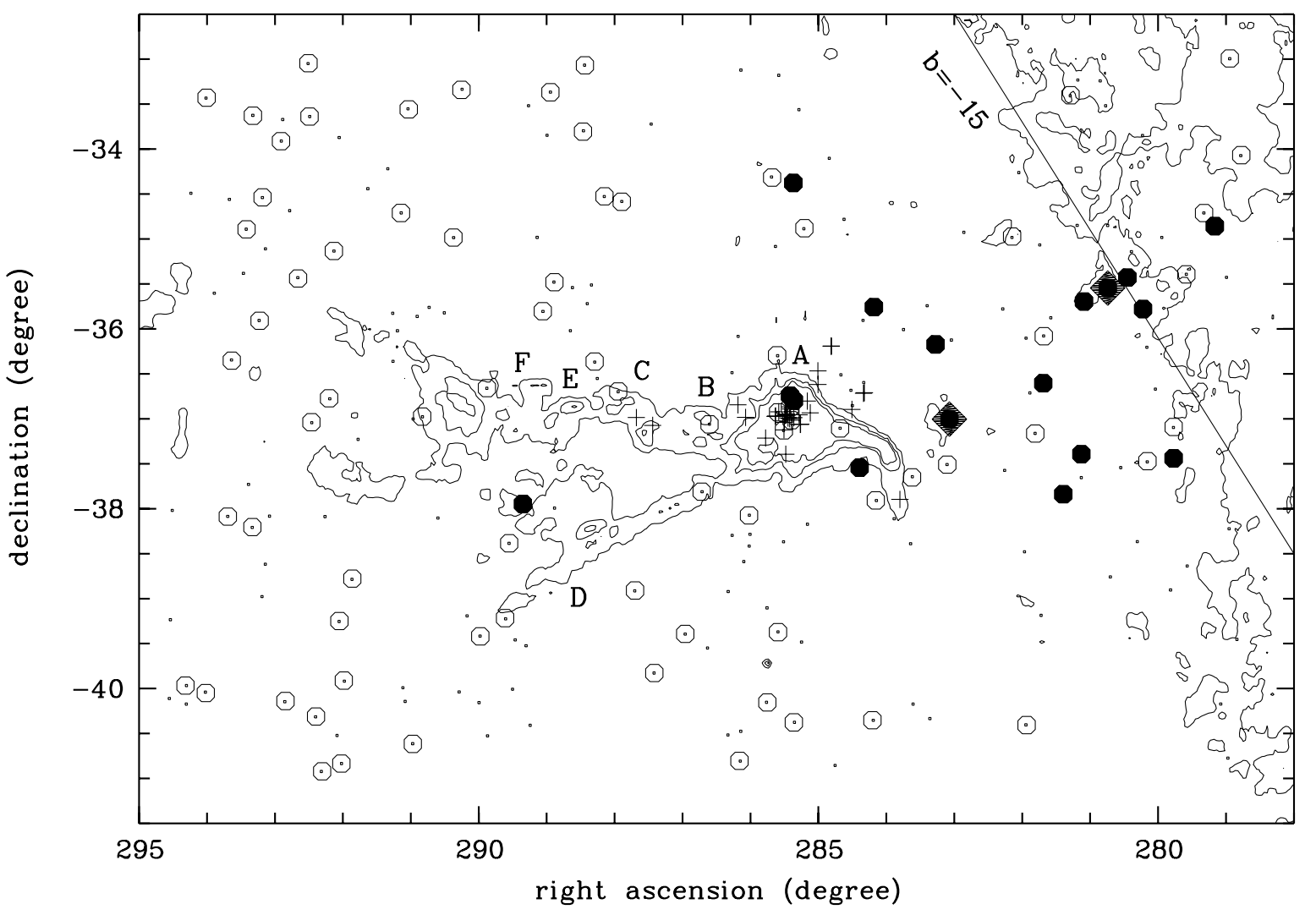

Fig. 1. Spatial distribution of RASS sources in CrA. We show all 206 RASS sources as dots superimposed on three contour levels of the IRAS $100 \mu \mathrm{m}$ map. RASS sources for which we did optical follow-up observations are circled (Table 3). Our new wTTS are shown by filled circles, new cTTS by filled diamonds (Table 4). Previously known PMS stars are indicated by plusses (Table 1). The clustering in the center is around the R CrA dark cloud. We also indicate by capital letters the condensations identified by Rossano (1978). Both new cTTS are located outside the main clouds, but near small cloud-lets. The galactic parallel $b=-15^{\circ}$ is indicated in the upper right corner

Finding charts for the new PMS stars can be found in Fig. 2, the optical spectra for our new TTS are shown in Fig. 3 (high-resolution spectra from CTIO, if available) and Fig. 4 (low-resolution spectra from ESO-1.52 m for the other new TTS). In Fig. 5, we also show the four spectra of previously suspected young stars obtained at the ESO-3.5 m-NTT.

As seen in Fig. 6, all new TTS show more lithium than ZAMS stars of the same spectral type, i.e. are younger than ZAMS; hence, they are pre-MS stars. There are some new M-type TTS in CrA, which have depleted more than half of their primordial lithium (lower right of Fig. 6). As for similar M-type TTS in Taurus, these objects could be classified as post-TTS.

\section{Optical and infrared photometry}

Broad band photometric observations in the VRI filters were carried out in 1997 from June 22 to 30 using the $0.9 \mathrm{~m}$ telescope of Cerro Tololo Interamerican Observatory (CTIO). The detector was a CCD Tektronix 2048 (CTIO \# 3) with pixel size $24 \mu \mathrm{m}$ and a readout noise of 3 to 5 electrons. The whole CCD was read out. Dome flat-field exposure sequences in each filter were taken typically before the beginning and upon the end of every night for flat field correction. Two or three different standard star fields from Landolt (1992) were also observed every night at different airmasses for the determination of atmospheric extinction, zero points and color transformation to the Johnson-Kron-Cousins standard system.

Raw CCD frames were bias subtracted and flat fielded using the IRAF package CCDRED. Sky flats were used, and the flat field variation across the final images was spot-checked and found to be negligible. The photometric 

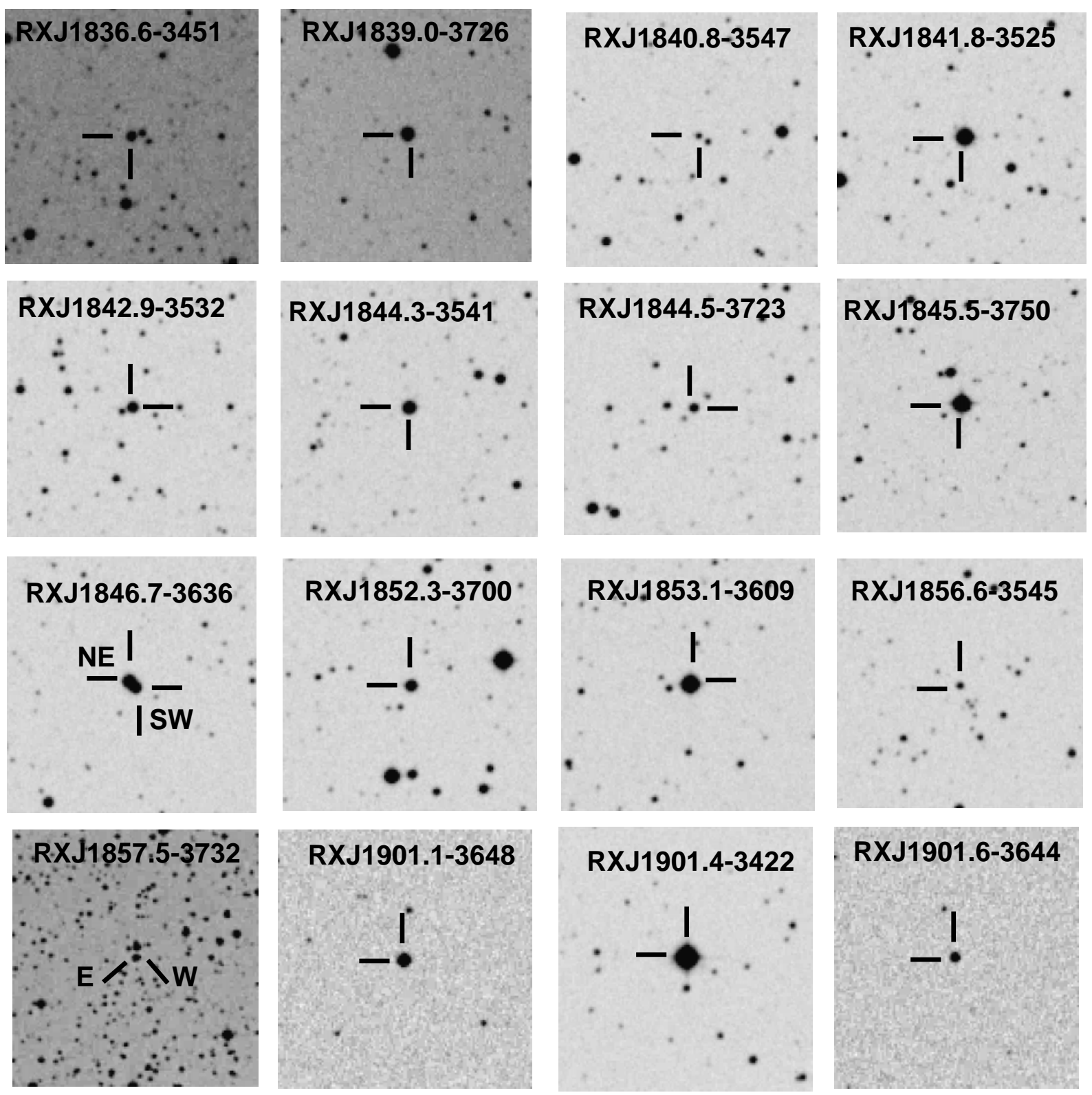

Fig. 2. Finding charts for the new PMS stars. We show $5^{\prime} \times 5^{\prime}$ DSS charts (north is up, east to the left) for all new PMS stars, with one exception, namely RXJ1917.4-3756, which is easy to identify as (by far) the brightest star in its field

solution for each night was determined using the IRAF tasks APPHOT and PHOTCAL. First, an aperture for each night was chosen by inspecting the reduced images from each night. The average FWHM over the run was about $1.3^{\prime \prime}$. To insure that all the light from a given star was in the aperture, a typical aperture for source extraction was taken as four times the FWHM or about $6^{\prime \prime}$. Annuli for background subtraction varied but were usually about $2^{\prime \prime}$ from inner diameter to outer diameter with an inner diameter of about $8^{\prime \prime}$. The background was taken as the median value within the annuli. An average of 30 calibration standards were observed each night at various airmasses. Photometric solutions for each night were determined by fitting the data to a color dependent airmass equation. The photometric errors dominate the Poisson errors in most cases.

Once the photometric solution for each night was determined, the target fields were examined. Aperture photometry was performed on all stars using the same aperture as was used for the standard stars on that night. The only exception to this was RXJ1857.5-3732. In this case, the two stars were separated by less than $4^{\prime \prime}$. Therefore we used the IRAF tool SUBSTAR to subtract one star from the image so we could accurately measure the instrumental magnitude of the other. The observed magnitude and colors were obtained by applying the instrumental magnitudes derived from the aperture to the photometric equation for the given night. Results are listed in Table 5. 

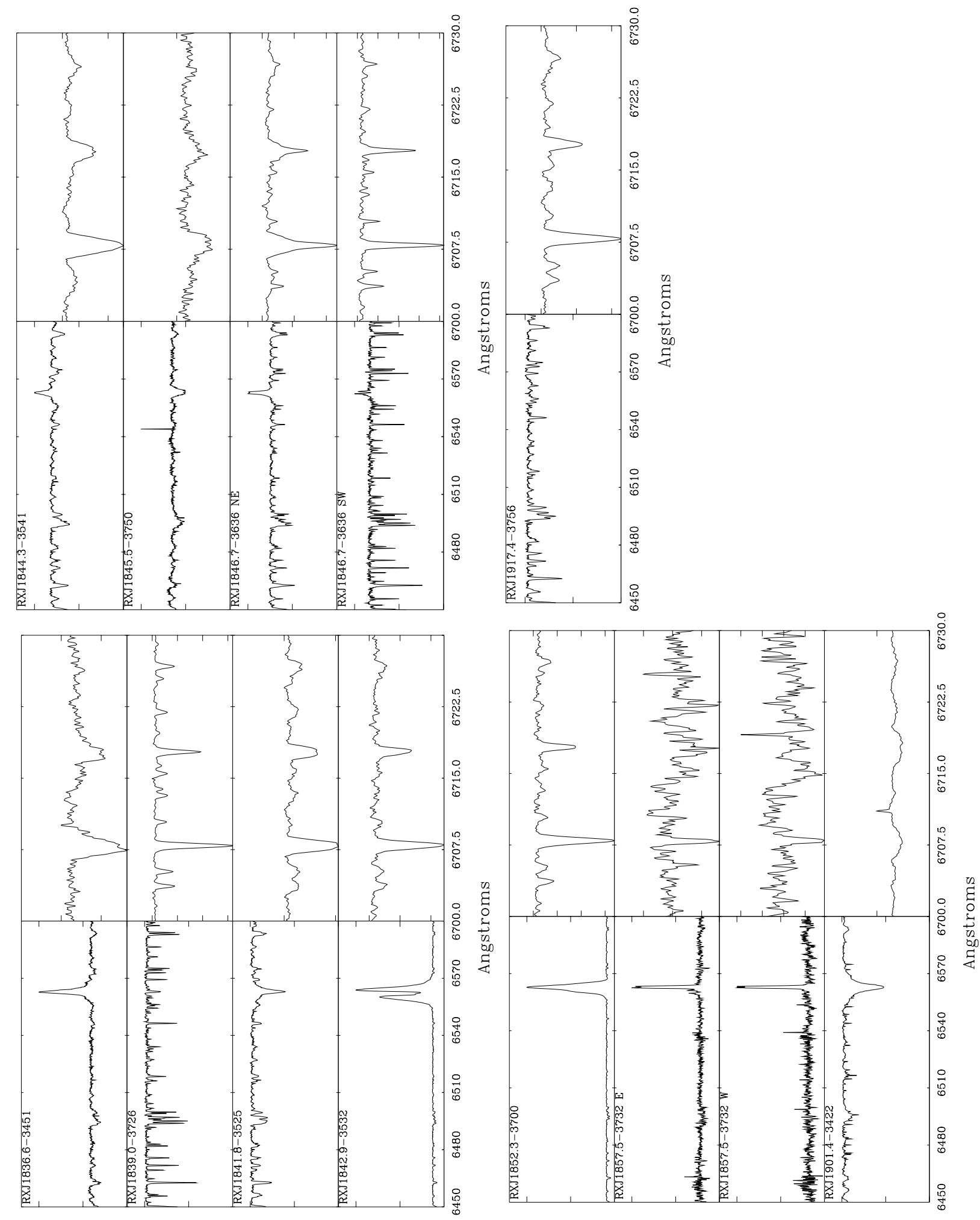

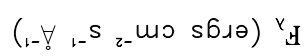

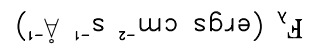

Fig. 3. High-resolution optical spectra of the new TTS. The spectra of new TTS observed with the CTIO 4-m echelle. The left panel shows a $250 \AA$ region including $\mathrm{H} \alpha$; the right panel shows $30 \AA$ including the $\mathrm{Li} \mathrm{I} \lambda 6707 \AA$ and Ca I $\lambda 6717 \AA$ lines. The left hand panels are scaled from 0 to $120 \%$ of the maximum flux, with ticks at the 50 and $100 \%$ levels. The right hand panels are scaled to show the shallow absorption profiles of the rapid rotators. In the right hand panels the ticks lie at $20 \%$ of the continuum level, with the top of the plot at the $120 \%$ level. If only a single tick is shown, the bottom of the panel is $80 \%$ of the maximum flux 

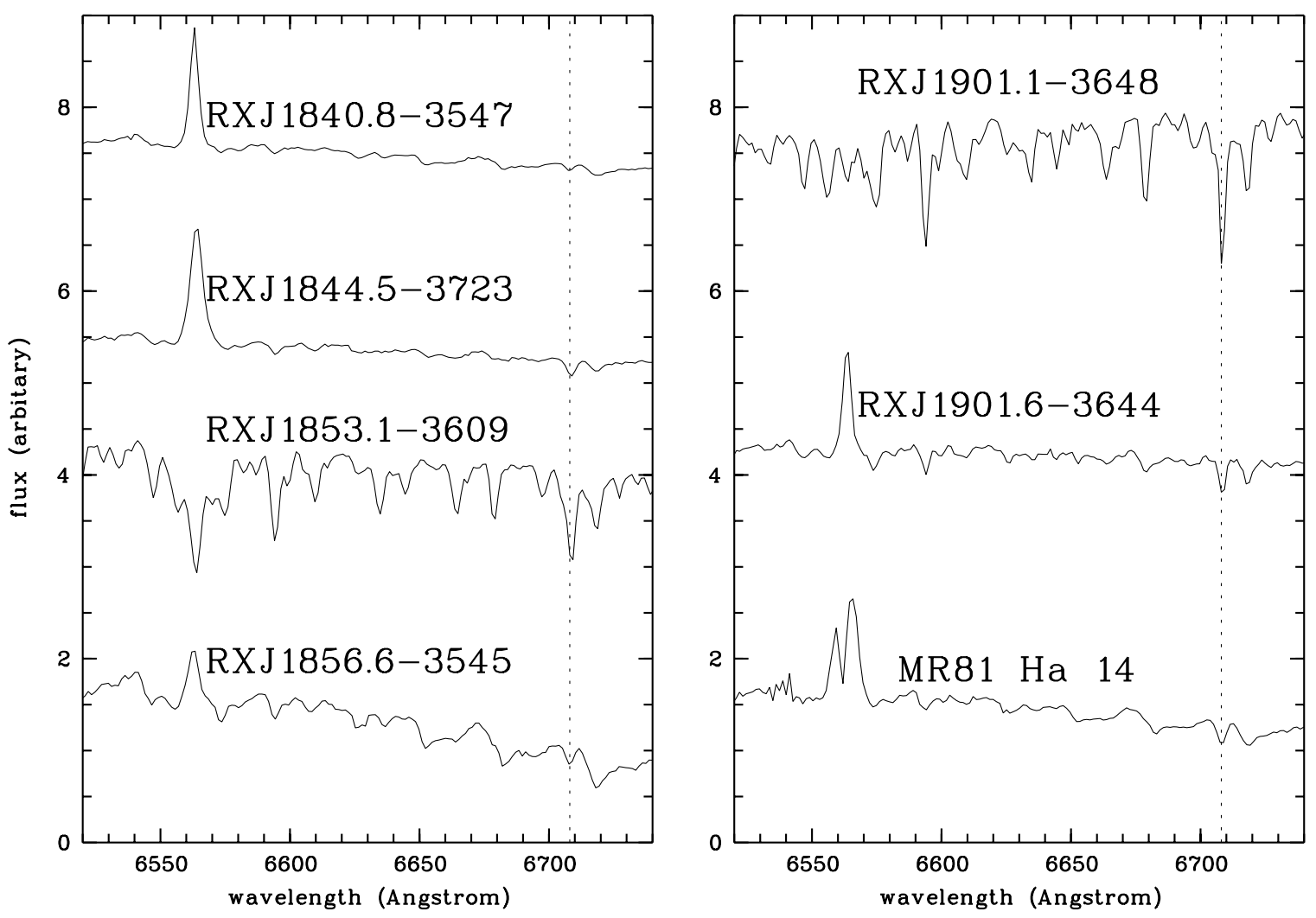

Fig. 4. Low-resolution optical spectra of the other new TTS. Spectra obtained at the ESO-1.52 m of those new TTS not shown in Fig. 3 as well as for MR81 $\mathrm{H} \alpha$ 14, also found to be wTTS. The dotted line shows the location of the lithium $6708 \AA$ line

The near-IR data were obtained using the CTIO InfraRed Imager, $\mathrm{CIRIM}^{6}$, on the $1.5 \mathrm{~m}$ telescope at CTIO. CIRIM is a $256 \times 256 \mathrm{HgCdTe}$ array. We observed at the f/13.5 focus, giving a plate scale of 0.65 arcsec per pixel. The data were obtained on the nights of 1998 July 8 to 14. All nights were photometric. The stellar point-spread function is well-sampled photometrically. Dome flats were taken at the start of each night. Flats were obtained with the dome illumination lamps both on and off, to determine the thermal contribution to the flat image. Standard stars, taken from the lists of Elias (1982) and the UKIRT faint standards (Casali \& Hawarden 1992), were observed hourly, at a full range of airmasses. Exposure times varied between 0.4 and 3 seconds, based on source magnitude, with 3 images coadded at each position. We observed the standard stars using a $2 \times 2$ raster, with 30 arcsec spatial offsets between positions. We observed the targets using exposure times between 0.4 and 20 seconds, based on expected source brightness, with 3 images coadded at each

\footnotetext{
${ }^{6}$ See Elston 1999 at

http://www.ctio.noao.edu/instruments/ir_instruments/cirim/ cirim.html
}

position. We observed using a $2 \times 3$ raster, with spatial offsets of 15 arcsec between frames.

We linearized the data using the IRAF routine IRLINCOR. All other processing was undertaken using our IDL-based CIRIM reduction package ${ }^{7}$. The images are divided by the appropriate normalized flat field image. The images in the raster pattern are median-filtered to determine the local sky image, which is then subtracted from each flattened image. The images in the raster pattern are then aligned by cross-correlating on the brightest sources in the image, and co-added. The image center and plate scale are determined by cross-correlating the images with stars in the USNO catalog (Monet et al. 1998). The photometric solution is determined by fitting the $\log$ of the standard star counts within a 12-pixel (7.8 arcsec) radius region as a linear function of the air mass. The solution is edited interactively to remove discrepant stars and/or points. The rms scatter in the photometric solutions is 1 to $2 \%$, and we take this to be our photometric precision. Magnitudes are determined by applying the photometric solution to the net counts

\footnotetext{
7 http://sbast3.ess.sunysb.edu/fwalter/CIRIM/cirim.html
} 
Table 4. New T Tauri stars in CrA. We list object number and ROSAT designation as in Tables 2 and 3, pre-main sequence type, spectral type, $\mathrm{H} \alpha$ and lithium equivalent widths (negative when in emission) from high-resolution spectra (unless given with colons), rotational velocity, helio-centric radial velocity, X-ray luminosity (in $130 \mathrm{pc}$ distance, calculated from counts, exposure and ECF listed in Table 2, 65 pc for TTS No. 82), and some remarks

\begin{tabular}{|c|c|c|c|c|c|c|c|c|c|}
\hline $\begin{array}{r}\text { No. } \\
\text { Table } 2\end{array}$ & $\begin{array}{l}\text { Object } \\
\text { designation }\end{array}$ & $\begin{array}{l}\text { PMS } \\
\text { type }\end{array}$ & $\begin{array}{l}\text { Spec } \\
\text { type }\end{array}$ & $\begin{array}{r}W_{\lambda}(\mathrm{H} \alpha) \\
{[\AA]}\end{array}$ & $\begin{array}{r}W_{\lambda}(\mathrm{Li}) \\
{[\AA]}\end{array}$ & $\begin{array}{r}v \cdot \sin i \\
{\left[\mathrm{~km} \mathrm{~s}^{-1}\right]}\end{array}$ & $\begin{array}{r}\text { RadVel } \\
{\left[\mathrm{km} \mathrm{s}^{-1}\right]}\end{array}$ & $\begin{array}{r}\log L_{\mathrm{X}} \\
{\left[\operatorname{erg~s}^{-1}\right]}\end{array}$ & Remarks \\
\hline 8 & RXJ1836.6-3451 & wTTS & M0 & -2.4 & 0.54 & 34.1 & -1.6 & 30.27 & \\
\hline 13 & RXJ1839.0-3726 & wTTS & K1 & +0.1 & 0.34 & 17.8 & -4.8 & 30.24 & $(1)$ \\
\hline 19 & RXJ1840.8-3547 & wTTS & M4 & -6.9 & $0.36:$ & & & 29.82 & \\
\hline 23 & RXJ1841.8-3525 & wTTS & G7 & +1.0 & 0.25 & 30.0 & -3.1 & 30.62 & $(2)$ \\
\hline 27 & RXJ1842.9-3532 & cTTS & $\mathrm{K} 2$ & -30.7 & 0.38 & 23.7 & -1.2 & 30.34 & $(3)$ \\
\hline 30 & RXJ1844.3-3541 & wTTS & K5 & -0.4 & 0.41 & 38.8 & -4.9 & 30.60 & \\
\hline 31 & RXJ1844.5-3723 & wTTS & M0 & $-5.3:$ & $0.52:$ & & & 30.59 & \\
\hline 36 & RXJ1845.5-3750 & wTTS & G8 & +0.8 & 0.24 & 54.6 & -1.3 & 30.81 & HD 173148 (1) \\
\hline 39 & RXJ1846.7-3636 NE & wTTS & K6 & -0.6 & 0.47 & 30.8 & -2.5 & 30.38 & $8^{\prime \prime}$ pair $(4)$ \\
\hline 39 & RXJ1846.7-3636 SW & wTTS & K7 & -0.2 & 0.43 & 17.5 & -2.6 & 30.38 & $8^{\prime \prime}$ pair (4) \\
\hline 53 & RXJ1852.3-3700 & cTTS & K3 & -33.8 & 0.51 & 21.8 & 0.0 & 30.41 & IRAS 18489-3703 (5) \\
\hline 55 & RXJ1853.1-3609 & wTTS & $\mathrm{K} 2$ & $+1.4:$ & 0.39: & & & 30.60 & HD 174656 \\
\hline 64 & RXJ1856.6-3545 & wTTS & M2 & $-1.6:$ & $0.25:$ & & & 29.78 & \\
\hline 68 & RXJ1857.5-3732 E & wTTS & M5 & -3.4 & 0.23 & 28.7 & -3.1 & 29.93 & close binary (4) \\
\hline 68 & RXJ1857.5-3732 W & wTTS & M6 & -4.7 & 0.42 & 11.9 & -2.9 & 29.93 & close binary (4) \\
\hline 80 & RXJ1901.1-3648 & wTTS & K4 & $+0.1:$ & $0.44:$ & & & 29.88 & VSS VIII-27 \\
\hline 82 & RXJ1901.4-3422 & wTTS & F7 & +2.2 & 0.09 & 57.6 & -3.3 & 29.77 & HD $176383(2)$ \\
\hline 85 & RXJ1901.6-3644 & wTTS & M0 & $-2.3:$ & $0.54:$ & & & 30.18 & VSS VIII-26 \\
\hline 135 & RXJ1917.4-3756 & wTTS & K2 & -0.9 & $0.48:$ & & & 30.61 & SAO 211129 \\
\hline
\end{tabular}

Notes: (1) Complex $\mathrm{H} \alpha$ profile. (2) Given the relatively early spectral type and relatively small lithium strength, this star could be either pre- or zero-age MS stars (see also Sect. 6.3). Its Hipparcos distance is $65 \mathrm{pc}, L_{\mathrm{X}}$ given for 65 pc. (3) Also observed with low resolution in 1995 and twice in 1996, when $W_{\lambda}(\mathrm{H} \alpha)$ varied from $-53.6 \AA$ to $-88.7 \AA$ from night to night. Double-peaked H $\alpha$ emission, also HeI and [OII] emission. (4) Visual pair; position given in Table 3 is for the primary, i.e. the slightly brighter star; not resolved spatially with ROSAT (the combined $L_{\mathrm{X}}$ is given). (5) Observed once in 1995 with low resolution and about the same $\mathrm{H} \alpha$ as given above for high-resolution spectrum, and five times with low resolution in 1996 with $W_{\lambda}(\mathrm{H} \alpha)$ from $-5.22 \AA$ to $-8.97 \AA$.

observed in a 12-pixel (7.8 arcsec) radius region. In the case of close binaries, we determined the total flux using a large extraction radius, and the relative magnitudes using smaller extraction radii. Typical uncertainties are $\pm 0.02 \mathrm{mag}$, but this degrades for the fainter targets. Results are listed in Table 5.

\section{6. $\mathrm{H}-\mathrm{R}$ diagram, ages, and masses}

Because we have a homogeneous and almost complete set of precise $V J H K$ photometric data, we estimate the absorption in the line-of-sight to the stars from the observed $V-J, V-H$, and $V-K$ colors and their intrinsic color indexes, which we know from their spectral types. For those few stars, for which $J H K$ is not available, we estimate the absorption from $V R I$ colors. Visual extinctions $A_{V}$ for all new TTS are listed in Table 5. Then, we can also estimate the bolometric luminosity $L_{\text {bol }}$ at an assumed distance of $130 \mathrm{pc}$, also listed in Table 5 .

We display the location of the new TTS in the H-R diagram in Fig. 7 together with tracks and isochrones from D'Antona \& Mazzitelli (1994). They yield rough estimates for ages and masses of our new TTS, which we also list in Table 5. It can been seen in Fig. 7 that the two visual pairs of TTS, RXJ1846 and RXJ1857, are both roughly co-eval. The ages of the new TTS range from less than one million years to a few tens of million years, not surprising given the wide spatial distribution. From that wide distribution on the sky around the CrA dark cloud, we have to conclude that the distances can show a similar spread, so that the ages and masses are uncertain. For the newly found young star RXJ1901.4-3422, whose parallax was measured by the Hipparcos satellite, we estimated its luminosity using the Hipparcos distance of $65 \mathrm{pc}$; it then falls on the 30 Myrs isochrone, just above the ZAMS; because its spectral type is late $\mathrm{F}$, it is difficult to classify it as either pre- or zero-age MS given its lithium. One more newly found Li-rich star, RXJ1901.1-3648, is also found near the ZAMS, using $130 \mathrm{pc}$ as distance, which may of course be a wrong distance. The latter one has spectral type K4 and clearly more lithium than Pleiades K4-type stars; it is located right on the cloud and its extinction is large $\left(A_{V} \simeq 3 \mathrm{mag}\right.$, Table 5$)$, hence it may be deep inside the dark cloud. 
Table 5. Photometric data for new T Tauri stars in CrA. We list object number as in Tables 2 to 4 . Values in brackets are errors for the last digit(s). When no error is given, data are taken from Simbad, GSC, or USNO, which are more uncertain (indicated by colons). For TTS No. 64, the JHK data are from the 2nd 2MASS data release. Bolometric luminosity are estimated for a distance of $130 \mathrm{pc}(65 \mathrm{pc}$ for TTS no. 82). Ages and masses are estimated by comparison with isochrones and tracks from D'Antona \& Mazzitelli (1994). The last object, RXJ1855.1-3754, is the TTS found in Neuhäuser et al. (1997), a ROSAT HRI source, not a RASS source, see Table 1

\begin{tabular}{lccccccccccc}
\hline $\begin{array}{l}\text { No. } \\
\text { Table 2 }\end{array}$ & $\begin{array}{c}B \\
\mathrm{mag}\end{array}$ & $\begin{array}{c}V \\
\mathrm{mag}\end{array}$ & $\begin{array}{c}V-R \\
\mathrm{mag}\end{array}$ & $\begin{array}{c}R-I \\
\mathrm{mag}\end{array}$ & $\begin{array}{c}K \\
\mathrm{mag}\end{array}$ & $\begin{array}{c}J-K \\
\mathrm{mag}\end{array}$ & $\begin{array}{c}H-K \\
\mathrm{mag}\end{array}$ & $\begin{array}{c}A_{V} \\
\mathrm{mag}\end{array}$ & $\begin{array}{r}\text { log } \\
L_{\mathrm{bol}} / L_{\odot}\end{array}$ & $\begin{array}{c}\text { age } \\
{[\mathrm{Myr}]}\end{array}$ & $\begin{array}{c}\text { mass } \\
{\left[M_{\odot}\right]}\end{array}$ \\
\hline 8 & $14.3:$ & $13.22(3)$ & $0.98(1)$ & $1.08(1)$ & $9.02(5)$ & $0.86(6)$ & $0.20(6)$ & 0.7 & -0.45 & 3.2 & 0.6 \\
13 & $12.0:$ & $10.81(1)$ & $0.50(2)$ & $0.50(1)$ & $8.51(2)$ & $0.62(3)$ & $0.14(3)$ & 0.2 & 0.04 & 10 & 1.3 \\
19 & $18.8:$ & $14.99(3)$ & $1.24(3)$ & $1.34(2)$ & $10.14(4)$ & $0.91(16)$ & $0.25(7)$ & 0.0 & -0.68 & 1.6 & 0.3 \\
23 & & $9.7:$ & & & $8.04(4)$ & $0.41(15)$ & $0.07(7)$ & 0.0 & 0.30 & 10 & 1.4 \\
27 & $12.3:$ & $12.28(3)$ & $0.70(1)$ & $0.71(1)$ & $8.23(3)$ & $1.35(5)$ & $0.51(5)$ & 1.5 & -0.04 & 10.0 & 1.2 \\
30 & & $11.3:$ & & & $8.38(3)$ & $0.71(5)$ & $0.18(5)$ & 0.1 & 0.07 & 1.0 & 0.9 \\
31 & $14.3:$ & $13.1:$ & & & $9.38(3)$ & $0.85(6)$ & $0.22(5)$ & 0.1 & -0.64 & 6.3 & 0.6 \\
36 & $10.0:$ & $9.2:$ & & & $7.18(3)$ & $0.44(5)$ & $0.10(5)$ & 0.3 & 0.73 & 4.0 & 1.5 \\
$39-\mathrm{NE}$ & & $11.16(3)$ & $0.73(2)$ & $0.66(1)$ & $7.89(3)$ & $0.72(5)$ & $0.11(5)$ & 0.4 & 0.25 & 0.5 & 0.7 \\
$39-\mathrm{SW}$ & & $11.43(2)$ & $0.24(1)$ & $0.66(1)$ & $7.89(3)$ & $0.72(5)$ & $0.11(5)$ & 0.5 & 0.20 & 0.5 & 0.6 \\
53 & $12.7:$ & $12.35(3)$ & $0.73(1)$ & $0.72(1)$ & $9.01(3)$ & $0.78(5)$ & $0.21(5)$ & 1.0 & -0.25 & 10 & 1.1 \\
55 & $11.0:$ & $9.6:$ & & & $7.31(3)$ & $0.61(5)$ & $0.12(5)$ & 0.1 & 0.46 & 2.0 & 1.7 \\
64 & $14.9:$ & $12.9:$ & & & $9.91(3)$ & $0.91(5)$ & $0.30(7)$ & 0.0 & -0.25 & 0.8 & 0.4 \\
$68-\mathrm{E}$ & & $15.56(3)$ & $1.2(1)$ & $1.5(1)$ & $10.53(2)$ & $0.96(4)$ & $0.20(3)$ & 0.0 & -0.91 & 2.5 & 0.2 \\
$68-\mathrm{W}$ & & $16.5(3)$ & $1.2(3)$ & $1.7(2)$ & $11.28(3)$ & $0.95(5)$ & $0.31(4)$ & 0.0 & -1.29 & 2.5 & 0.1 \\
80 & & $15.85(4)$ & $1.29(3)$ & $1.52(2)$ & & & & 3.0 & -0.86 & 40 & 0.7 \\
82 & $8.7:$ & $8.2:$ & & & $7.07(3)$ & $0.23(5)$ & $0.05(5)$ & 0.0 & 0.30 & 30 & 1.2 \\
85 & $15.5:$ & $14.27(3)$ & $1.17(2)$ & $1.23(2)$ & & & & 1.1 & -0.70 & 7.9 & 0.6 \\
135 & $10.8:$ & $9.9:$ & & & $7.54(3)$ & $0.69(5)$ & $0.14(5)$ & 0.1 & 0.36 & 3.2 & 1.6 \\
\hline RXJ1855.1-3754 & $13.05(5)$ & $0.62(3)$ & $0.57(3)$ & & & & 0.3 & -0.82 & 40 & 0.7 \\
\hline
\end{tabular}

Our new Li-rich ROSAT counterparts show a larger age spread than the Li-rich EO counterparts (Walter et al. 1997). This is probably due to the larger spread in distances among the ROSAT stars compared to the EO stars, because we investigated a larger area on the sky using the RASS as compared Walter et al. (1997) who used EO pointed observations centered on the CrA dark cloud.

The two new cTTS, RXJ1842.9-3532 and RXJ1852.33700 , both appear to be $\sim 10$ Myrs old, which is relatively old for cTTS. This result only holds if they are indeed at $\sim 130 \mathrm{pc}$ as assumed for calculating their $L_{\mathrm{bol}}$, which may be unrealistic, because they are located on the line-of-sight to two small cloud-lets, both off the main CrA dark cloud. On the other hand, there are also some other cTTS with ages around 10 Myrs, namely TW Hya and HD 98800, for which the Hipparcos parallaxes are known, so that they could be placed correctly into the $\mathrm{H}-\mathrm{R}$ diagram. Both two new CrA cTTS show only small IR excess emission at $J H K$, and one of them, RXJ1852.3-3700, is an IRAS source.

The two TTS with the latest spectral types in our sample are RXJ1857.5-3732 E and W with M5 and M6, respectively. They are both certainly young because of the strong lithium absorption. Hence, they are most certainly members of the CrA dark cloud, i.e. at 130 pc. Then, in the $\mathrm{H}-\mathrm{R}$ diagram, they lie near the borderline between stars and brown dwarfs with masses around $\sim 0.1$ to $\sim 0.2 M_{\odot}$. At first glance, it appears surprising to find such lowmass objects among RASS sources. Previously, several borderline objects and even brown dwarfs were detected as X-ray sources, but only in very deep pointed observations (Neuhäuser \& Comerón 1998; Neuhäuser et al. 1999; Neuhäuser \& Comerón 2000). However, our two RASS detected mid- to late-M dwarfs form a close pair, i.e. only one unresolved RASS X-ray source. Given their optical/IR luminosities, spectral types, and typical $L_{\mathrm{X}} / L_{\mathrm{bol}}$ ratios, they would not have been detected individually in the RASS.

\section{Kinematics}

Because CrA is not part of the Gould Belt, the young stars surrounding the CrA dark cloud most certainly are associated with the CrA association. They could have formed near the present locations, where the gas has dispersed since then. In that case, these young stars should be somewhat older than the on-cloud TTS. Alternatively, if the CrA cloud has not shrunk, the off-cloud young stars could have dispersed out of the cloud, either slowly (then, the outermost stars would again be older than the on-cloud stars) or, at least in some cases, have higher velocities. In the latter case, one would expect at least a few young run-away TTS ejected from the dark cloud. For checking 


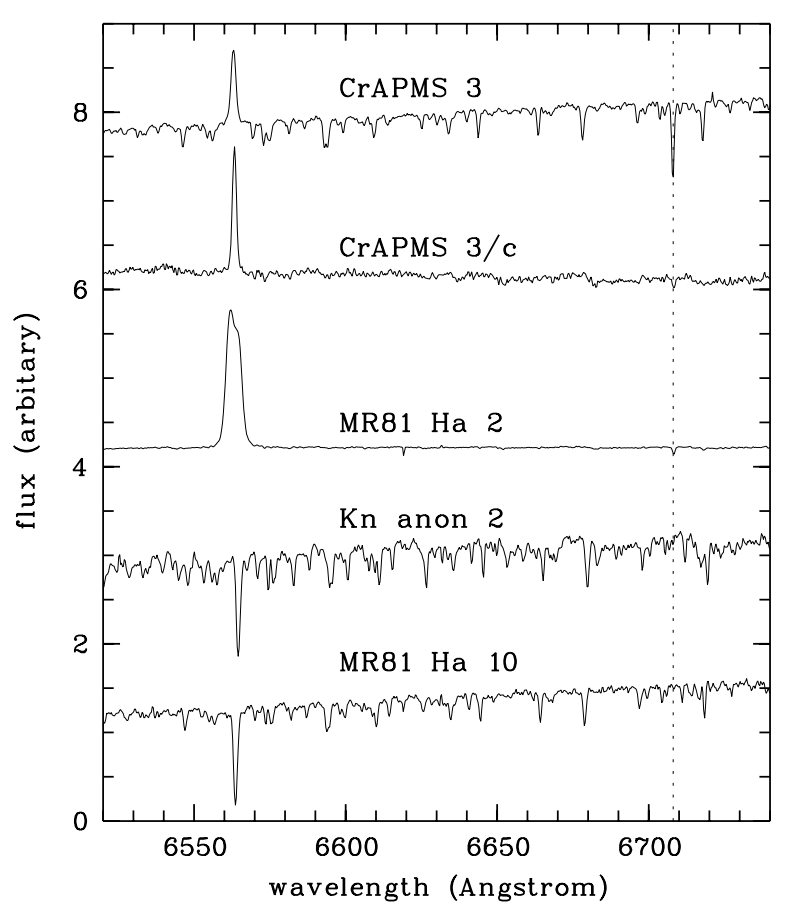

Fig. 5. Low-resolution optical spectra of previously suspected young stars. Spectra obtained at the ESO-3.5 m-NTT of stars suspected to be young prior to the ROSAT mission, namely CrAPMS 3/c (new wTTS), MR81 H $\alpha 2$ (new cTTS), Kn anon 2, MR81 H $\alpha 10$ (no lithium, i.e. both non-TTS), and for comparison we also show CrAPMS 3 (wTTS). The dotted line shows the location of the lithium $6708 \AA$ line

these possibilities, we need to investigate proper motions and radial velocities.

\subsection{Proper motions}

In order to examine the kinematical state of the stars in the R CrA association, we searched for proper motions for all the stars in Tables 1 and 3 in the Hipparcos (ESA 1997), PPM (Röser \& Bastian 1991; Bastian et al. 1993; Röser et al. 1994), ACT (Urban et al. 1997); TRC (Høg et al. 1998), Tycho2 (Høg et al. 2000), and STARNET (Röser 1996) proper motion catalogs. Altogether we could identify 24 stars in these catalogs, including nine stars known already before the ROSAT mission, nine TTS newly identified here, as well as four ZAMS and two $\mathrm{dKe} / \mathrm{dMe}$ stars. Note that $\mathrm{R}$ CrA itself is not listed; it is included in the Hipparcos catalog (HIP 93449), but no meaningful solution for its astrometric parameters could be derived. Proper motions are given in Table 6. For stars present in more than one catalog we usually adopted the most precise proper motion determination, unless it was in conflict with values from other catalogs. All proper
Table 6. Proper motions for CrA TTS. Listed are objects from Tables 1 to 4, which are included in at least one of the proper motion catalogues Hipparcos (HIP), PPM, ACT, TRC (T), STARNET (S) or Tycho2 (T2). All proper motions refer to the Hipparcos astrometric system

\begin{tabular}{|c|c|c|c|}
\hline object & cat. no. & 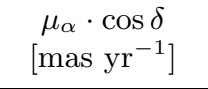 & $\begin{array}{c}\mu_{\delta} \\
{\left[\text { mas } \mathrm{yr}^{-1}\right]}\end{array}$ \\
\hline \multicolumn{4}{|c|}{ Known and suspected CrA member stars before ROSAT: } \\
\hline $35720-3643$ & T2 74211242 & $5.3 \pm 2.6$ & $-29.1 \pm 2.7$ \\
\hline $185801-3$ & S 74211040 & $13 \pm 6.1$ & $-27 \pm 6.1$ \\
\hline HR $7169^{a}$ & Т 74212294 & $10.1 \pm 2.6$ & $-28.8 \pm 2.6$ \\
\hline HR $7170^{a}$ & 212295 & $7.0 \pm 2.6$ & $-15.5 \pm 2.6$ \\
\hline $\mathrm{S}$ CrA & S 7421213 & $10 \pm 4.6$ & $-26 \pm 4.6$ \\
\hline $\mathrm{CoD}-37^{\circ} 13022$ & T2 74211890 & $6.6 \pm 2.5$ & $-27.6 \pm 2.6$ \\
\hline HD 176386 & HIP 9 & $1.6 \pm 1.8$ & $-26.7 \pm 0.9$ \\
\hline V702 CrA & T2 7 & $4.9 \pm 1.8$ & $-26.1 \pm 1.8$ \\
\hline SAO 210888 & HIP 93689 & $4.5 \pm 1.6$ & $-28.6 \pm 0.8$ \\
\hline \multicolumn{4}{|c|}{ T Tauri stars newly discovered with ROSAT (RXJ...): } \\
\hline & & $0.6 \pm 2.6$ & $-29.1 \pm 2.6$ \\
\hline & T2 7 & $7.0 \pm 1.9$ & $-25.1 \pm 1.9$ \\
\hline $1842.9-3532$ & S 7415696 & $3 \pm 4.8$ & $-27 \pm 4.8$ \\
\hline 541 & S 7 & $-8 \pm 4.8$ & $-32 \pm 4.8$ \\
\hline 18 & T2 7915531 & $4.6 \pm 2.0$ & $-25.9 \pm 2.0$ \\
\hline $.3-3700$ & S 74 & $1 \pm 5.8$ & $-28 \pm 5.8$ \\
\hline 1853.1-3609 & T2 7420774 & $2.9 \pm 1.8$ & $-24.6 \pm 1.7$ \\
\hline $1901.4-3422^{b}$ & HIP 93412 & $9.9 \pm 1.5$ & $-46.8 \pm 1.0$ \\
\hline 1917.4-3756 & T2 7918222 & $8.4 \pm 1.5$ & $-25.8 \pm 1.5$ \\
\hline \multicolumn{4}{|c|}{ Other stars (RXJ...): } \\
\hline & $\mathrm{T}$ & $-2.5 \pm 2.8$ & $-6.4 \pm 2.9$ \\
\hline 1911.5 & T2 74182446 & $13.3 \pm 1.8$ & $-15.7 \pm 1.9$ \\
\hline 1915.7-3321 & S 74271333 & $-3 \pm 5.1$ & $-5 \pm 5.1$ \\
\hline $1921.4-3459^{c}$ & HIP 95149 & $78.9 \pm 4.1$ & $-108.9 \pm 2.5$ \\
\hline 1928.5-3508 & HIP 95753 & $-16.3 \pm 2.1$ & $-14.4 \pm 1.4$ \\
\hline 1936.0-4002 & T2 7936832 & $-4.9 \pm 2.4$ & $7.8 \pm 2.4$ \\
\hline
\end{tabular}

(a) The visible double star HR $7169 / \mathrm{HR} 7170$ is represented by two entries in the Hipparcos Catalogue (HIP 93368 and HIP 93371) with two individual component solutions. The solution quality is classified as fair; however the proper motion errors are much smaller in TRC, which we adopt here.

(b) Hipparcos distance is $65 \pm 5 \mathrm{pc}$, i.e. foreground to the cloud.

(c) RXJ 1921.4-3459 has an acceleration solution in the Hipparcos Catalogue.

motions were transformed to the Hipparcos astrometric system (ICRS) before comparison.

Positions and proper motion diagrams for stars in Table 6 are shown in Fig. 8. The mean proper motion of the $\mathrm{R}$ CrA member stars seems to be very well defined. All except maybe one (HR 7170) of the member stars known before ROSAT (including the late B-type stars listed at the end of Table 1, whose membership was not clear before) show very similar proper motions, and all except two of the newly identified TTS 


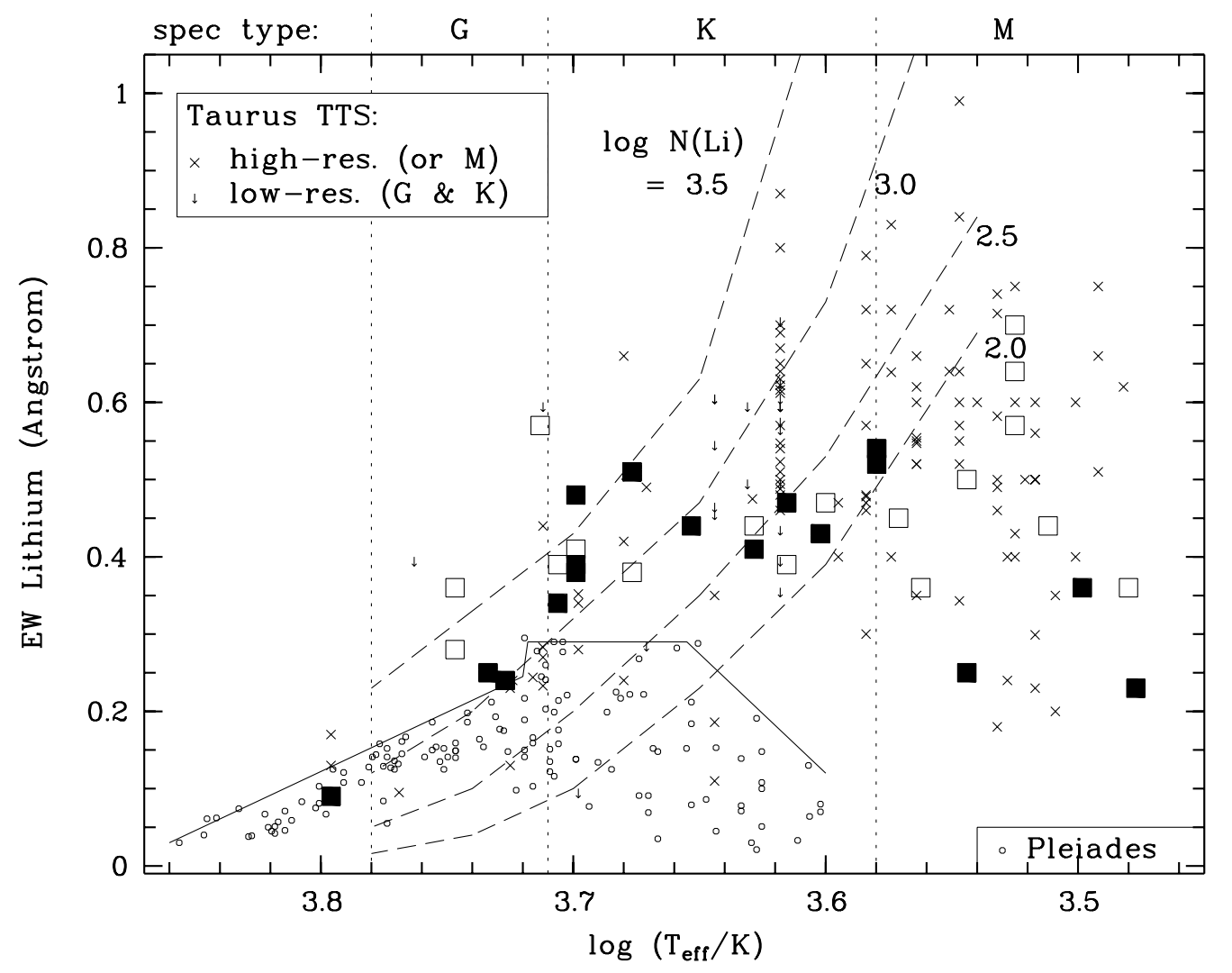

Fig. 6. Lithium versus effective temperature. We plot the lithium equivalent width $W_{\lambda}(\mathrm{Li})$ versus the effective temperature $T_{\text {eff }}$ for the previously known TTS in CrA (Table 1, open squares), the newly identified PMS stars (Table 4, full squares), bona-fide Taurus TTS (crosses and arrows, see Neuhäuser et al. 1997 for references), and the Pleiades as dots (Soderblom et al. 1993; García López et al. 1994). We converted spectral types to $T_{\text {eff }}$ following Bessell $(1979,1991)$. Also shown are lithium iso-abundance lines (as dashed lines) for $\log g=4.5$ from Pavlenko \& Magazzù (1996). Stars with more lithium than ZAMS stars of the same spectral type, i.e. stars which lie above the upper envelope (solid line) of the Pleiades lithium data, are younger than ZAMS, i.e. PMS stars. Because late F- and G-type stars do not significantly burn lithium during the ZAMS phase, the three full squares in the lower left could be either PMS or ZAMS stars

nicely follow this trend. In contrast to this, the stars classified as ZAMS or $\mathrm{dKe} / \mathrm{dMe}$ form a kinematically much more inhomogeneous distribution. The mean proper motion for all 15 likely members of the association is $\left(\mu_{\alpha} \cos \delta, \mu_{\delta}\right)=(5.5,-27.0) \mathrm{mas} \mathrm{yr}^{-1}$. The largest part of this motion is simply the reflex of the solar motion, which is $\left(\mu_{\alpha} \cos \delta, \mu_{\delta}\right) \approx(7.1,-23.7){\text { mas } \mathrm{yr}^{-1}}^{-1}$ at $19^{\mathrm{h}} 00^{\mathrm{m}},-37^{\mathrm{o}} 00^{\prime}$ and a distance of $130 \mathrm{pc}$, and $\left(\mu_{\alpha} \cos \delta, \mu_{\delta}\right) \approx(5.6,-24.0)$ mas yr$^{-1}$ for $18^{\mathrm{h}} 45^{\mathrm{m}}$ and the same declination and distance values as above. Thus the slight difference between the mean proper motion in right ascension of the eight $\mathrm{R}$ CrA member stars known before $\operatorname{ROSAT}\left[\left(\mu_{\alpha} \cos \delta, \mu_{\delta}\right)=(7.0,-27.5) \mathrm{mas} \mathrm{yr}^{-1}\right]$ and the seven newly identified member TTS $\left[\left(\mu_{\alpha} \cos \delta, \mu_{\delta}\right)\right.$ $=(3.9,-26.4)$ mas $\left.r^{-1}\right]$ is partly a projection effect reflecting the fact that the new TTS are located at slightly lower right ascension.

\subsection{Space velocities}

We calculated space velocities for those stars with measured radial velocities (taken from Table 1 for six stars and from Table 4 for another six stars) and corrected them for the influence of galactic rotation (Fig. 9). The solar motion has also been subtracted, although this does not change the relative space velocities between stars, in contrast to galactic rotation or projection effects.

With the exception of HD 176386, which shows a discordant motion in the $U$-direction, all calculated space velocities are very similar. The mean $(U, V, W)$ values are $(4.8,-2.1,-2.7) \mathrm{km} \mathrm{s}^{-1}$ for the stars known before ROSAT (excluding HD 176386 for taking the mean of the $U$-velocities) and $(4.4,-2.2,-0.7) \mathrm{km} \mathrm{s}^{-1}$ for the new TTS, i.e. no systematic differences seem to be present. There could still be a small difference in the $W$-velocities, 
but the number of stars is so small that this is maybe not significant.

The velocity dispersion is indeed very low, $\left(\sigma_{U}, \sigma_{V}, \sigma_{W}\right)=(1.4,1.2,1.8) \mathrm{kms}^{-1}$ for the whole sample, which excludes the ejection mechanism (so-called run-away TTS) as major source for off-cloud TTS, because they should have discrepant velocities. The velocity dispersion is highest in the $W$-direction, and taking also into account that the positions of the stars form a broader distribution in the $Z$-direction $\left(\sigma_{Z}=4.9 \mathrm{pc}\right.$, or $\sigma_{Z}=4.0 \mathrm{pc}$ if the far off lying RXJ1917.4-3756 is excluded) than in the $Y$-direction $\left(\sigma_{Y}=1.9 \mathrm{pc}\right)^{8}$ this could be interpreted in terms of stars oscillating around the galactic plane. It simultaneously would explain why no PMS stars were found in the opposite direction of the R CrA cloud: the stars already reached the largest distance from the galactic plane and are currently near their turning point, consistent with their $W$-velocities being close to zero. One complete oscillation around the galactic plane would last $\sim 10^{8} \mathrm{yrs}$, but need not be finished by now for this scenario to be true. The off-cloud stars are currently located on average at $z=-35 \mathrm{pc}$, while the cloud is at $z=-39 \mathrm{pc}$. The stars which now appear to be off-cloud and the cloud itself, given their current locations and velocities and tracing their paths back in time, would have been at the same location $\sim 310^{7}$ yrs ago. The stars located outside of the current cloud borders are on average $\sim 10$ Myrs old, i.e. older than those inside the cloud, which supports the cloud oszillation scenario. However, the details and exact time-scales depend on the unknown total cloud mass. A very similar interpretation could also explain the positions and the motions of the stars found south of the Taurus clouds (Neuhäuser et al. 1997; Frink et al. 1997).

Lépine \& Duvert (1994) suggested that high velocity cloud impacts could trigger star formation, and that subsequently to the impact clouds and stars could be separated from each other due to different friction during the passage through the galactic plane. If this is true, the new TTS found outside the dark cloud today could very well have been born inside the molecular cloud. The fact that the observed velocity dispersions are so low supports this scenario. A typical proper motion uncertainty of 3 mas $\mathrm{yr}^{-1}$ and an assumed distance uncertainty of $15 \mathrm{pc}$ translate into a combined uncertainty of $\approx 3 \mathrm{~km} \mathrm{~s}^{-1}$ for the $V$ and $W$ components of the space velocity. The observed velocity dispersions are found to be even lower, so that this is consistent with a very small intrinsic dispersion of the velocities.

\footnotetext{
8 This can also be seen in Fig. 1 , where the $Z$-direction is more or less perpendicular to the $b=-15^{\circ}$ line, whereas the $Y$-direction is parallel to this line (the new TTS lying north of the central cloud is RXJ1901.4-3422, a young foreground star, see Sect. 7.3).
}

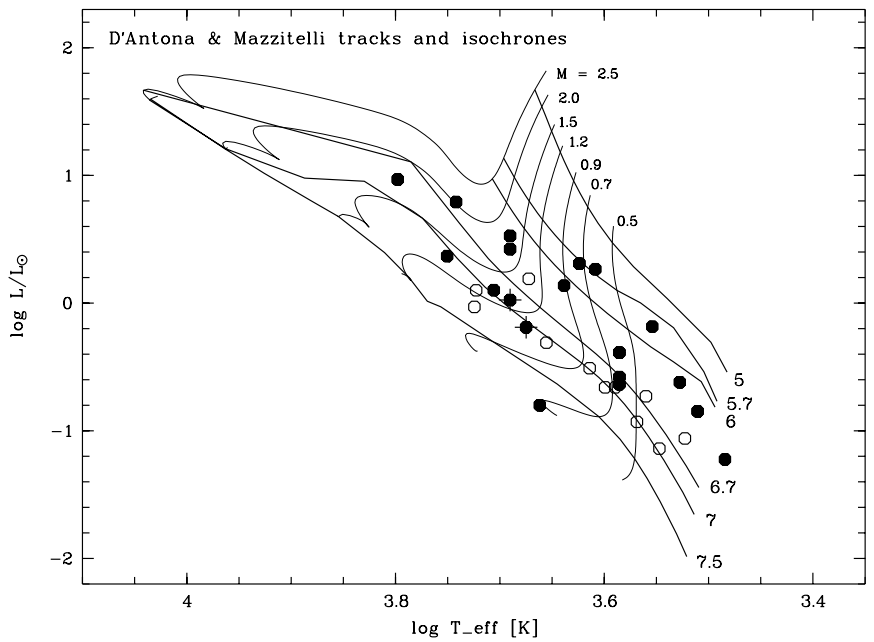

Fig. 7. H-R diagram with the newly found young stars. Bolometric luminosity versus effective temperature for all newly found Li-rich ROSAT counterparts (from Table 4, all filled symbols) and for Li-rich EO counterparts (Walter et al. 1997, open symbols), compared to tracks and isochrones from D'Antona \& Mazzitelli (1994) for ages (given on a log scale) and masses (given in $M_{\odot}$ ). The two newly found cTTS are marked by a plus sign; surprisingly, they are relatively old, namely $\sim 10$ Myrs old (at the assumed distance of $130 \mathrm{pc}$ )

\subsection{Stars with discordant proper motions}

There are three stars among the sample of pre-ROSAT $\mathrm{R}$ CrA members and newly identified TTS with discordant proper motions from the mean.

HR 7170 belongs to a possibly quadruple system, and therefore the proper motion determination is highly problematic (see footnote to Table 6). Given the fact that the TRC proper motion of HR 7169 is consistent with kinematical membership to the $\mathrm{R} \mathrm{CrA}$ association and that HR 7170 presumably belongs to the same system, it is likely that the different TRC proper motion of HR 7170 reflects orbital motions within the system. Although the kinematical membership of HR 7170 could not be proven directly, it still should be considered an R CrA association member. The stars HR 7169 and HR 7170 lie in a cavity in the CO distribution (Loren 1979). This suggests similar distances to the stars and cloud. Additionally, optical images show an extensive reflection nebulosity surrounding the pair.

The two other stars are the newly identified TTS RXJ1844.3-3541 and RXJ1901.4-3422. The case for RXJ1844.3-3541 is not clear. According to its spectral type and lithium line strength, it is clearly a young TTS, and also its radial velocity is consistent with membership. Either the proper motion from STARNET maybe in error or the star could have been ejected from the cloud. Comparing its proper motion with the association mean, it would move towards the south-west relative to the 

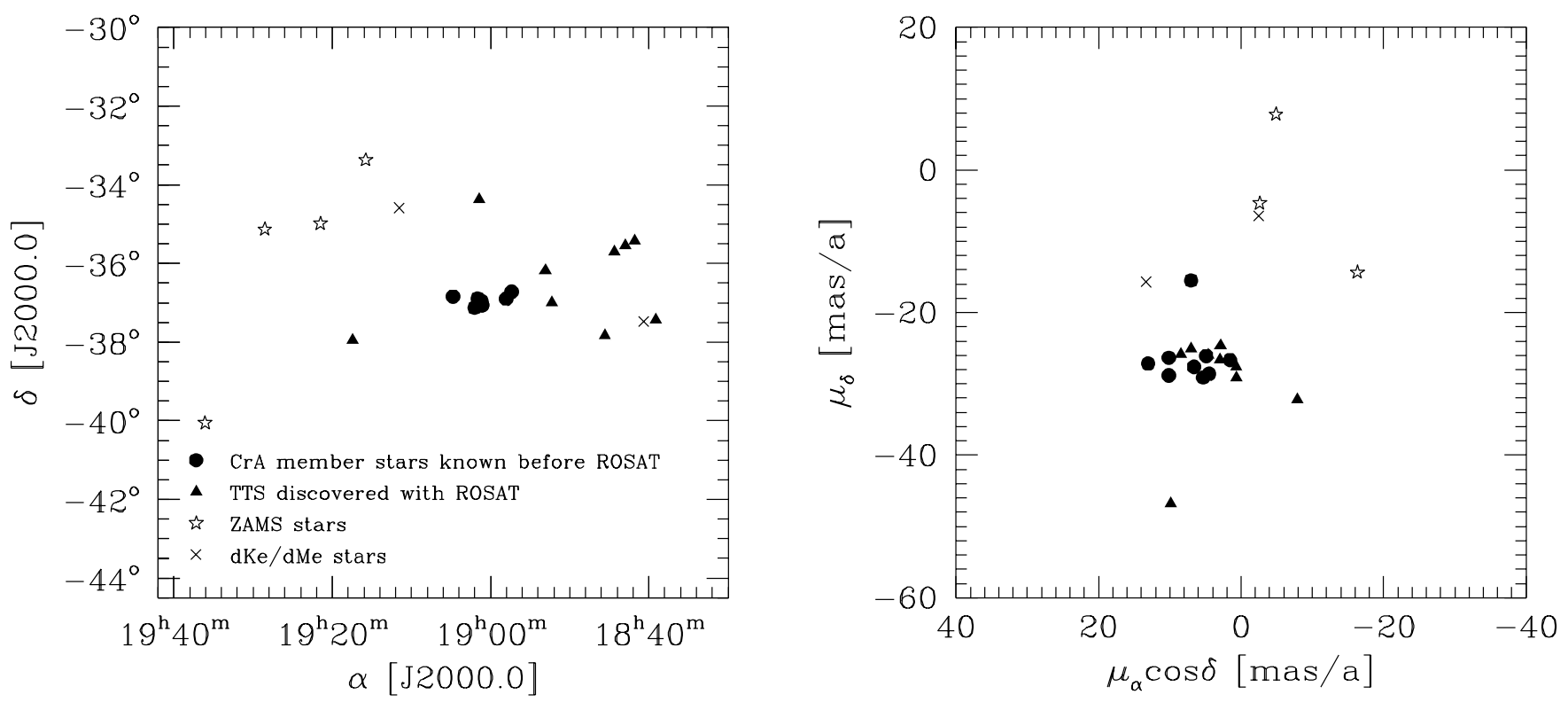

Fig. 8. Positions (left panel) and proper motions (right panel) of all the stars in Table 6, i.e. with proper motions available. The mean proper motion of R CrA member stars is well defined by most of the previously known stars (including early-type as well as late-type stars) and newly identified young stars in this region. Note that the proper motions of the stars classified as TTS are much more uniformly distributed than the proper motions of the stars classified as ZAMS or $\mathrm{dKe} / \mathrm{dMe}$
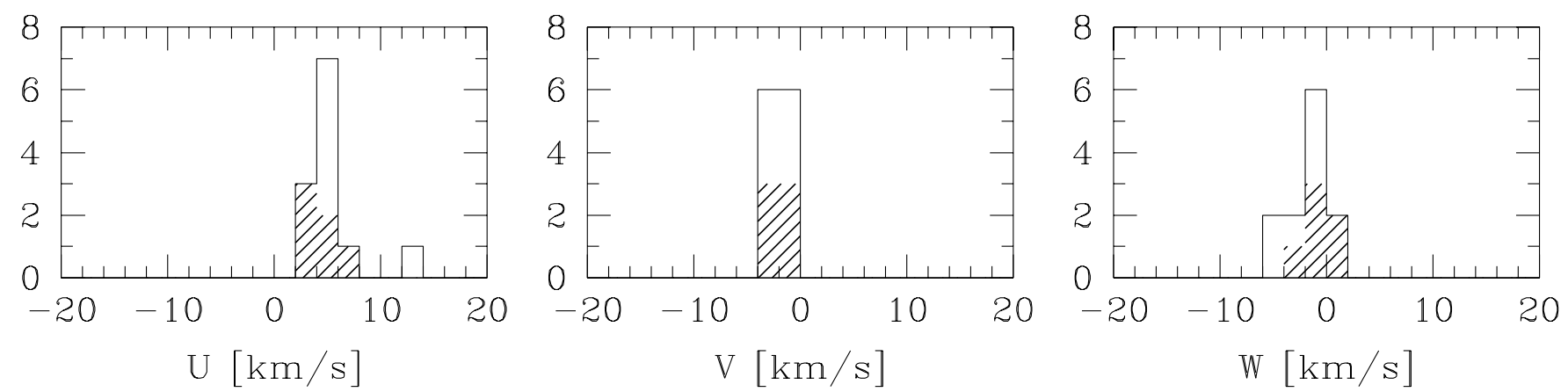

Fig. 9. Space velocities for all likely R CrA member stars (six stars known before ROSAT and six newly identified TTS shown hatched) with known proper motions and radial velocities. The effects of differential galactic rotation and the reflex of the solar motion were eliminated using IAU standard values for all constants. A distance of $130 \mathrm{pc}$ has been assumed for all stars. $U$ points in the direction of the galactic center, $V$ in the direction of galactic rotation and $W$ towards the north galactic pole. The velocity dispersions are very small

cloud, but it is located north-west of the cloud, so that it probably was not ejected from the R CrA cloud.

For RXJ1901.4-3422 the classification as TTS is doubtful. At a late-F spectral type, even Pleiades ZAMS stars have not yet burned significantly lithium, so that classification as either pre-MS or ZAMS stars is difficult. Because its lithium line is stronger than its nearby calcium line, we classify the star as TTS. The Hipparcos parallax of $65 \pm 5$ pc clearly places it foreground to the $\mathrm{R}$ CrA cloud, which explains its large proper motion, and it is the only star located clearly north of the $\mathrm{R}$ CrA cloud. We conclude that RXJ1901.4-3422 is most likely not an association member, but young.

\section{Completeness of our survey}

In Fig. 10, we compare the complete $\log N-\log S$ curve with those for stellar, extra-galactic, and unidentified sources. At a PSPC count rate threshold of $0.1 \mathrm{cts} \mathrm{s}^{-1}$, $90 \%$ of the RASS sources have optical counterparts, most of them being stars. At lower count rates the fraction of unidentified sources increases from $\sim 30 \%$ at $0.07 \mathrm{cts} \mathrm{s}^{-1}$ to more than $50 \%$ at 0.02 cts s$^{-1}$, a level at which all curves flatten because of the incompletness of the RASS itself. Above a PSPC count rate of $0.03 \mathrm{cts} \mathrm{s}^{-1}$ we have optically identified 75 among 136 RASS X-ray sources (i.e. $55 \%$ ), most of them being stars. The small number of extra-galactic object identified at this threshold 


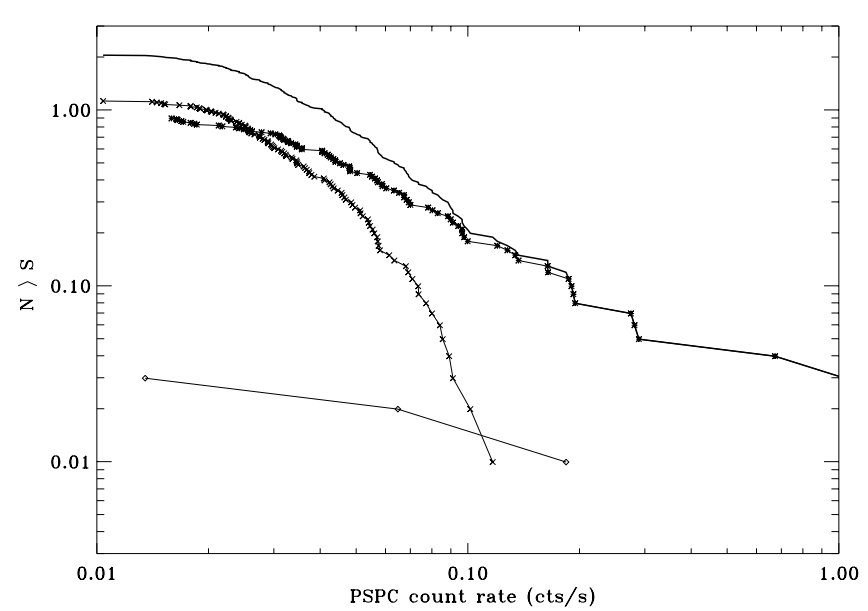

Fig. 10. The $\log N-\log S$ curves in the CrA region. Number of X-ray sources per $\mathrm{deg}^{2}$ detected by ROSAT as a function of PSPC count rate for all RASS sources listed in Table 2 (solid bold line), for the optically identified stellar (star symbols) and extra-galactic (diamonds) populations as well as for the unidentified sources $(x)$

Table 7. X-ray source density. Surface density of stellar X-ray sources observed on $\left(\rho_{\text {On }}\right)$ and off $\left(\rho_{\text {Off }}\right) \mathrm{R}$ CrA cloud, model predictions $\left(\rho_{\text {model }}\right)$ as well as observed-prediction ratio as a function of PSPC count rate threshold $S$ in $\mathrm{cts} / \mathrm{s}$

\begin{tabular}{clllll}
\hline$S$ & $\rho_{\text {On }}$ & $\rho_{\text {off }}$ & $\rho_{\text {model }}$ & $\rho_{\text {On }} / \rho_{\text {model }}$ & $\rho_{\text {Off }} / \rho_{\text {model }}$ \\
\hline 0.10 & 0.179 & 0.167 & 0.113 & 1.58 & 1.47 \\
0.05 & 0.626 & 0.414 & 0.273 & 2.29 & 1.51 \\
0.03 & 1.073 & 0.682 & 0.482 & 2.22 & 1.41 \\
\hline
\end{tabular}

(only two) is simply due to our follow-up observation strategy. According to the results of Guillout (1996) and Zickgraf et al. (1997), we conclude that the extra-galactic population in the $\mathrm{R}$ CrA region is likely to account for about $25 \%$ of the unidentified X-ray sources detected above $0.03 \mathrm{cts} \mathrm{s}^{-1}$, the rest (i.e. $20 \%$ ) probably being optically faint active $\mathrm{K}$ - and M-type stars.

We now focus on the identified stellar population and define two sub-regions within our field, namely the oncloud region (from $\alpha=18 \mathrm{~h} 56 \mathrm{~m}$ to $19 \mathrm{~h} 24 \mathrm{~m}$ and from $\delta=-38^{\circ}$ to $-36^{\circ}$, i.e. $\left.14 \mathrm{deg}^{2}\right)$ and the off-cloud region (complementary to the on-cloud region, i.e. $112 \mathrm{deg}^{2}$ ).

We have plotted in Fig. 11 the observed on-cloud and off-cloud stellar $\log N-\log S$ curves as well as the predictions of the stellar X-ray population model from Guillout et al. (1996). Computations were run for $|b|=15^{\circ}$ and $l=180^{\circ}$ although the galactic longitude is irrelevant at the RASS sensitivity. Results are summarized in Table 7.

First we note that at any PSPC count rate the on-cloud stellar density is significantly higher (by a factor 2) compared to the model predictions, as expected for a region with ongoing star formation. On the other hand, we expect that the off-cloud $\log N-\log S$ curve lies within $15 \%$ of the model prediction, which is clearly not the case.

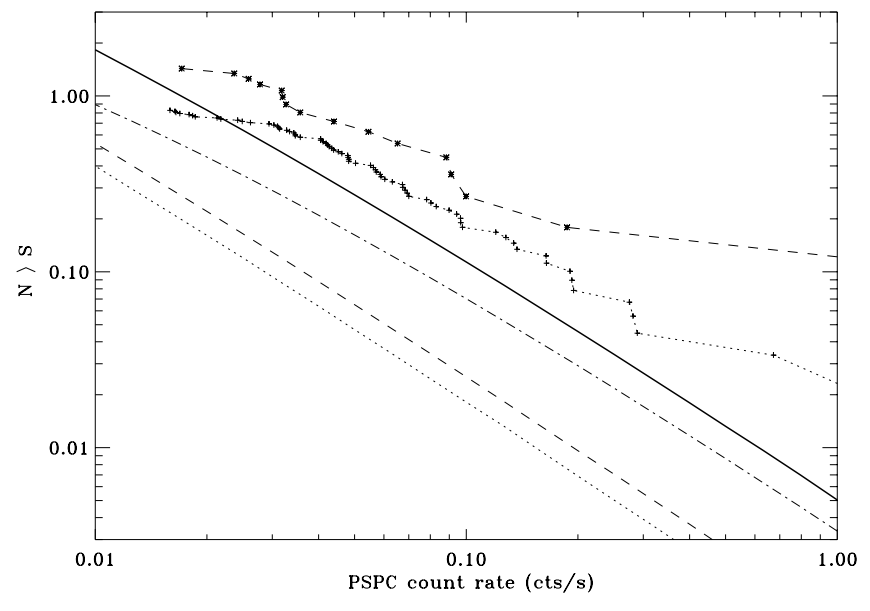

Fig. 11. The stellar $\log N-\log S$ curves in the CrA region. Number of stellar X-ray sources per $\mathrm{deg}^{2}$ optically identified on-cloud (star symbol) and off cloud (plus symbol) regions as well as predictions from stellar X-ray population model. Bold curve is for all model age bins, dash-dotted line for stars younger than 150 Myrs, dashed line for stars from 150 to 1000 Myrs old and dotted line for stars older than 1000 Myrs

In order to check the relevance of the model prediction, we compare with the so-called RasTyc sample (Guillout et al. 1999), a sample of all objects included in both RASS and Tycho, i.e. the largest sample of stellar X-ray sources with homogeneous and accurate data constructed so far. In order to account for the magnitude and X-ray flux limited biases of of RasTyc, we ran a specific model optically limited at $10.5 \mathrm{mag}$ (plus $20 \%$ of stars down to $11.5 \mathrm{mag}$ ). We then compared the expected number of stars per $\mathrm{deg}^{2}$ with the observed one computed in two regions extending $10^{\circ}$ wide all around the sky and centered at $|b|=15^{\circ}$. At a PSPC count rate threshold of $0.03 \mathrm{cts} \mathrm{s}^{-1}$, there are 1819 RasTyc stars detected within these two regions amounting to $6946 \mathrm{deg}^{2}$, i.e. 0.26 stars per $\mathrm{deg}^{2}$. At this level, the model predicts a stellar surface density of 0.23 stars per $\mathrm{deg}^{2}$, in very good agreement with the observations. We are thus confident that the theoretical $\log N-\log S$ curves plotted in Fig. 11 give a good estimation of the ambient galactic plane stellar population at the $\mathrm{R}$ CrA cloud galactic latitude.

We then conclude that in the region surrounding the CrA molecular cloud our observations reveal $\sim 40 \%$ excess of stellar X-ray sources with respect of a "pure" galactic plane population (see Table 7). According to the expected contribution of extra-galactic sources to the unidentified population, $40 \%$ is a lower limit on the excess. Such excesses were also detected around other star forming regions (see Neuhäuser 1997 and references therein). However, contrary to some other star forming regions like Lup-Sco-Cen (Guillout et al. 1998a,b), the Gould Belt can hardly be an explanation because of the position of the 
CrA molecular cloud projected well below the Gould Belt plane.

Also around the Chamaeleon clouds (Alcála et al. 1995) and south of the Taurus clouds (Neuhäuser et al. 1997), many new pre-MS stars were found, although there is no Gould Belt in that directions. As far as the Chamaeleon off-cloud TTS are concerned, Mizuno et al. (1998) found new, previously unknown, small cloud-lets near one third of the off-cloud TTS, which may be the birth places of those seemingly off-cloud TTS. If one can explain off-cloud TTS around the CrA and Cha clouds by cloud-lets rather than by the Gould Belt, at least some of the Lup-Sco-Cen, and Orion off-cloud TTS may also have originated in such small cloud-lets, as originally proposed for the Chamaeleon off-cloud TTS by Feigelson (1996).

The question now is whether we found all young, i.e. coronally active stars (inside and) around the CrA dark cloud. This can be investigated by optical follow-up observations of additional unidentified X-ray sources found in deep ROSAT PSPC and HRI pointed observations (Walter et al., in preparation).

Whether all young stars were found among all RASS sources can be investigated in the following way: Sterzik et al. (1995) have shown that it is possible to pre-select TTS candidates from the RASS using four criteria, namely the two hardness ratios, the X-ray count rate, and the optical magnitude of the nearest (if any) counterpart (within, say, 40"). Then, TTS candidates are those RASS sources which resemble best previously known RASS-detected bona-fide TTS according to the same properties. The parameter which describes how well a particular RASS source resembles the typical TTS properties is called discrimination probability $P$, described in detail in Sterzik et al. (1995).

In Fig. 12, we plot the number of CrA RASS sources per discrimination probability $P$, namely for PMS stars, otherwise active stars, other objects, and unidentified RASS sources. If we would have pre-selected TTS candidates using the Sterzik et al. (1995) method, i.e. if we would have done optical follow-up observations only for RASS sources with a discrimination probability of, say, $P \geq 0.45$, we would have obtained a high success rate by loosing only one TTS.

Now, for a discrimination probability of, say, $P=0.5$, the reliability (rel) of the TTS candidate selection is 0.45 , based on the classified sub-sample. The reliability number gives the fraction of real TTS (real according to our spectroscopy) among those X-ray sources with discrimination probability above some threshold, e.g. $P \geq 0.5$. The fraction of lost unidentified TTS is 0.17 , which is the number of real TTS to be expected (according to their discrimination probability values) among those X-ray sources not observed by optical spectroscopy. Because there is a total of $N=46$ sources with $P \geq 0.5$ and 160 below

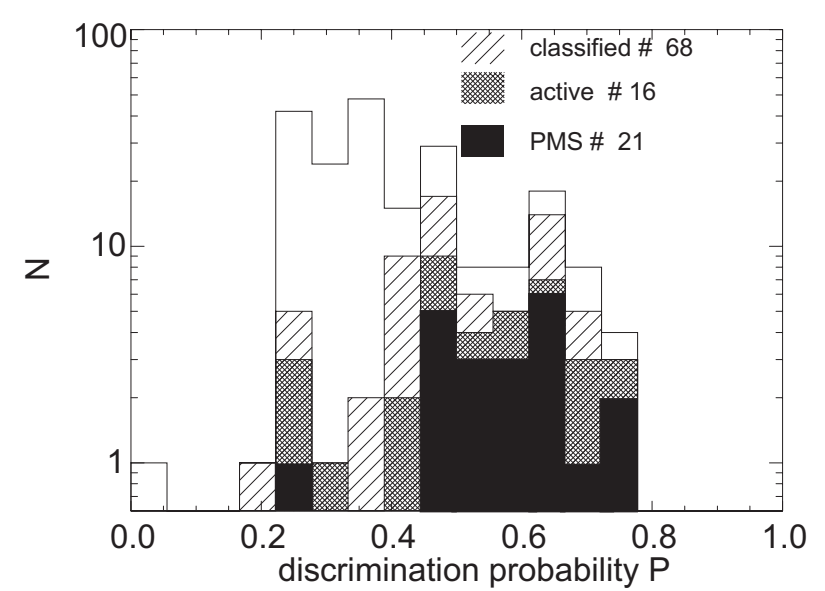

Fig. 12. Completeness of our follow-up observations. This histogram shows the number of X-ray sources per discrimination probability $\mathrm{P}$ for PMS stars (17 RASS sources identified with new PMS stars plus four RASS sources identified with previously known PMS stars), otherwise coronally active stars (nine ZAMS stars plus seven dKe/dMe stars), 68 X-ray sources identified with other objects, and all 206 RASS sources in CrA

this threshold, the expected number of TTS hidden in the RASS sample is

$$
\leq N(P \geq 0.5) \cdot \mathrm{rel}+N(P<0.5) \cdot \operatorname{loss}=48 \text {. }
$$

Hence, there should be a total of $\leq 48 \mathrm{X}$-ray sources with TTS as true counterparts (with an X-ray flux above the RASS flux limit). The lower limit can be found when considering only those RASS sources which are unclassified, but do have an optical counterpart; there are $\tilde{N}=46$ such sources with $P \geq 0.5$ and 46 with $P<0.5$. Hence, there should be

$$
\geq \tilde{N}(P \geq 0.5) \cdot \operatorname{rel}+\tilde{N}(P<0.5) \cdot \operatorname{loss}=29
$$

RASS sources with TTS as true counterparts. Of those 29 to 48 sources, 21 are identified as such, namely as previously known or newly found TTS. The remaining eight to 27 still unknown TTS should be found among as yet unidentified RASS sources. According to Fig. 10, most of those missing TTS identifications are due to the magnitude limit in the catalogs used here (e.g. the GSC).

\section{Summary}

In this data paper, we presented ground-based optical and infrared follow-up observations of previously unidentified ROSAT All-Sky Survey X-ray sources in and around the R CrA star forming cloud. We identified two new cTTS and 17 new wTTS having stronger lithium absorption than ZAMS stars of the same spectral type. Radial velocities and proper motions of most of these new TTS are consistent with the previously known CrA TTS, but the new objects are distributed more widely in space. Compared to a galactic model, this sample of ROSAT TTS constitutes an excess of young stars, more than expected at this 
galactic latitude. We estimated that there should be in total 29 to 48 RASS detectable TTS in CrA, of which 8 to 27 hitherto unknown TTS are still hidden among as yet unobserved ROSAT Survey sources. Similar to the two new cTTS found to be located projected onto two small cloud-lets, the seemingly off-cloud wTTS found here may have formed in small cloud-lets, which have dispersed since they formed those stars. For this scenario as well as for the ejection model (run-away TTS), a larger than observed velocity dispersion would be expected. The space motions of the on-cloud and off-cloud TTS are consistent with a scenario in which cloud and stars oscillate around the galactic plane, with a very small intrinsic velocity dispersion. This could possibly be the result of a high-velocity cloud impact triggering star formation, as advocated by Lépine \& Duvert (1994). However, alternative scenarios cannot be ruled out given the accuracy of the kinematical data available at present.

Acknowledgements. We would like to thank the staff at ESO La Silla and CTIO for their help as well as George Leussis for his significant contribution in the reduction of the photometric data. We are gratefull to our referee, Dr. Bruce Wilking, for many helpful comments, which improved the content of this paper. This research has made use of the Simbad database, operated at CDS, Strasbourg, France. This publication makes use of data products from the Two Micron All Sky Survey, which is a joint project of the University of Massachusetts and the Infrared Processing and Analysis Center/California Institute of Technology, funded by the National Aeronautics and Space Administration and the National Science Foundation. The ROSAT project is supported by the German Government (BMBF/DLR) and the Max-Planck-Society. RN wishes to acknowledge financial support from the Bundesministerium für Bildung und Forschung through the Deutsche Zentrum für Luft- und Raumfahrt e.V. (DLR) under grant number 50 OR 0003.

\section{References}

Adams F., Lada C., Shu F., 1987, ApJ 312, 788

Alcalá J.M., Krautter J., Schmitt J.H.M.M., et al., 1995, A\&AS 114, 109

Alcalá J.M., Covino E., Torres G., Sterzik M.F., Pfeiffer M.J., Neuhäuser R., 2000, A\&A 353, 186

André P., Montmerle T., ApJ 420, 837

Barrado y Navascues D., 1998, A\&A 339, 831

Bastian U., Röser S., Yagudin L.I., et al., 1993, PPM Star Catalogue, Vols. III \& IV. Spektrum Akademischer Verlag, Heidelberg

Bessell M.S., 1979, PASP 543, 589

Bessell M.S., 1991, AJ 101, 662

Briceño C., Hartmann L., Stauffer J.R., Gagné M., Stern S., 1997, AJ 113, 740

Cambrésy L., 1999, A\&A 345, 965

Casali M.M., Hawarden T.G., 1992, JCMT-UKIRT Newsletter 4, 33

Casey B.W., Mathieu R.D., Vaz L.P.R., Anderson J., Suntzeff N.B., 1998, AJ 115, 1617
Chen H., Grenfell T.G., Myers P.C., Hughes J.D., 1997, ApJ 478, 295

Covino E., Alcalá J.M., Allain S., Bouvier J., Terranegra L., Krautter J., 1997, A\&A 328, 187

D’Antona F., Mazzitelli I., 1994, ApJS 90, 467

Dame T.M., Ungerechts H., Cohen R.S., 1987, ApJ 322, 706

ESA, 1997, The Hipparcos and Tycho Catalogues, ESA SP-1200

Elias J.H., Frogel J.A., Matthews K., Neugebauer G., 1982, AJ 87,1029

Feigelson E.D., 1996, ApJ 468, 306

Feitzinger J.V., Stüwe J.A., 1984, A\&AS 58, 365

Frink S., Röser S., Neuhäuser R., Sterzik M.F., 1997, A\&A 325,613

Gaposchkin A., Greenstein J.L., 1936, Harvard Obs. Bull., No. 904,8

García López R.J., Rebolo R., Martín E.L., 1994, A\&A 282, 518

Glass I.S., Penston M.V., 1975, MNRAS 172, 227 (GP75)

Guillout P., 1996, Ph.D., Observatoire Astronomique de Strasbourg, Université Louis Pasteur, France

Guillout P., Sterzik M.F., Schmitt J.H.M.M., Motch C., Egret D., Voges W., Neuhäuser R., 1998a, A\&A 334, 540

Guillout P., Sterzik M.F., Schmitt J.H.M.M., Motch C., Neuhäuser R., 1998b, A\&A 337, 113

Guillout P., Schmitt J.H.M.M., Egret D., Voges W., Motch C., Sterzik M.F., 1999, A\&A 351, 1003

Harju J., Haikala L.K., Mattila K., Mauersberger R., Booth R.S., Nordh H.L., 1993, A\&A 278, 569

Herbig G.H., 1978, "The post T Tauri stars". In: Mirzoyan L. (Hrsg.) Problems of Physics and Evolution of the Universe. Academy of Science of Armenia, Erevan, p. 171

Herbig G.H., Bell K.R., 1988, Third Catalog of Emission-Line Stars of the Orion Population. Lick Obs. Bull. 1111 (HBC)

Høg E., Kuzmin A., Bastian U., et al., 1998, A\&A 335, L65

Høg E., Fabricius C., Makarov V.V., et al., 2000, A\&A 355, L27

Hoffleit D., 1982, The Bright Star Catalog. 4th ed., Yale University Observatory, New Haven

Knacke R.F., Strom K.M., Strom S.E., Young E., Kunkel W., 1973, ApJ 179, 847

Koyama K., Hamaguchi K., Ueno S., Kobayashi N., Feigelson E.D., 1996, PASJ 48, L87

Landolt A.U., 1992, AJ 104, 340

Lépine J.R.D., Duvert G., 1994, A\&A 286, 60

Loren R.B., 1979, ApJ 227, 832

Marraco H.G., Rydgren A.E., 1981, AJ 86, 62 (MR81)

Miller G.E., Scalo J.M., 1979, ApJS 41, 513

Mizuno A., Hayakawa T., Yamaguchi N., et al., 1998, ApJ 507, L83

Monet D., Bird A., Canzian B., et al., 1998, USNO-A2.0, A Catalog of Astrometric Standards, U.S. Naval Observatory Flagstaff

Montmerle T., Koch-Miramond L., Falgaronne E., Grindlay J., 1983, ApJ 269, 182

Motch C., Guillout P., Haberl F., et al., 1998, A\&AS 132, 341

Neuhäuser R., 1997, Sci 276, 1363

Neuhäuser R., Preibisch Th., 1997, A\&A 322, L37

Neuhäuser R., Brandner W., 1998, A\&A 330, L29

Neuhäuser R., Comerón F., 1998, Sci 282, 83 
Neuhäuser R., Comerón F., 2000, Coronal activity of brown dwarfs. In: Rebolo R. (ed.), 11th Cambridge Workshop on Cool Stars, Stellar Systems, and the Sun (in press)

Neuhäuser R., Sterzik M.F., Schmitt J.H.M.M., Wichmann R., Krautter J., 1995, A\&A 297, 391

Neuhäuser R., Thomas H.-C., Danner R., Peschke S., Walter F.M., 1997, A\&A 318, L43

Neuhäuser R., Torres G., Sterzik M.F., Randich S., 1997, A\&A 325,647

Neuhäuser R., Briceño C., Comerón F., et al., 1999, A\&A 343, 883

Olano C.A., 1982, A\&A 112, 195

Patten B., 1998, The low-mass membership of the R CrA T association. In: Donahue R.A., Bookbinder J.A. (eds.), 10th Cambridge Workshop on Cool Stars, Stellar Systems, and the Sun, CD-1755

Pavlenko Ya P., Magazzù A., 1996, A\&A 311, 961

Pöppel W., 1997, Fund. Cos. Phys. 18, 1

Röser S., 1996, IAU Symp. 172, 481

Röser S., Bastian U., 1991, PPM Star Catalogue, Vols. I \& II. Spektrum Akademischer Verlag, Heidelberg

Röser S., Bastian U., Kuzmin A., 1994, A\&AS 105, 301

Rossano G.S., 1978, AJ 83, 234

Schmitt J.H.M.M., Fleming T.A., Giampapa M.S., 1995, ApJ 450, 392

Soderblom D.R., Jones B.F., Balachandran S., et al., 1993, AJ 106, 1059

Sterzik M.F., Durisen R., 1995, A\&A 304, L9
Sterzik M.F., Alcalá J.M., Neuhäuser R., Schmitt J.H.M.M., 1995, A\&A 297, 418

Stocke J.T., Liebert J., Gioia I.M., et al., 1983, ApJ 273, 458

Taylor K.N.R., Storey J.W.V., 1984, MNRAS 209, 5P

Torres G., 1985, A\&AS 59, 449

Urban S.E., Corbin Th.E., Wycoff G.L., 1997, The ACT Reference Catalog, U.S. Naval Observatory, Washington DC

Walter F.M., 1986, ApJ 306, 573

Walter F.M., Matthews L.D., 1997, Nat 389, 358

Walter F.M., Wolk S.J., Neuhäuser R., 1996, Nat 379, 233

Walter F.M., Brown A., Mathieu R.D., Myers P.C., Vrba F.J., 1988, AJ 96, 297

Walter F.M., Vrba F.J., Wolk S.J., Mathieu R.D., Neuhäuser R., 1997, AJ 114, 1544

Wichmann R., Covino E., Alcalá J.M., Krautter J., Allain S., Hauschildt P.H., 1999, MNRAS 307, 909

Wilking B.A., Lada C.J., Young E.T., 1984, ApJ 340, 823

Wilking B.A., Taylor K.N.R., Storey J.W.V., 1986, AJ 92, 103

Wilking B.A., Greene T.P., Lada C.J., Meyer M.R., Young E.T., 1992, ApJ 397, 520

Wilking B.A., McCaughrean M.J., Burton M.G., Giblin T., Rayner J.T., Zinnecker H., 1997, AJ 114, 2029

Zickgraf F.-J., Thiering I., Krautter J., et al., 1997, A\&AS 123, 103

Zimmermann H.U., Becker W., Belloni T., et al., 1994, EXSAS Users's Guide, MPE Report 257, ROSAT Scientific Data Center, Garching 